\title{
A Spherical Harmonic Analysis of the Ooty Wide Field Array (OWFA) Visibility Signal
}

\author{
Suman Chatterjee ${ }^{1,2 \star}$, Somnath Bharadwaj ${ }^{1,2} \dagger$ \\ ${ }^{1}$ Department of Physics, Indian Institute of Technology Kharagpur, Kharagpur - 721 302, India. \\ ${ }^{2}$ Centre for Theoretical Studies, Indian Institute of Technology Kharagpur, Kharagpur - 721 302, India.
}

9 November 2018

\begin{abstract}
Considering redshifted 21-cm intensity mapping with the upcoming OWFA whose field of view subtends $\sim 57^{\circ}$ in the N-S direction, we present a formalism which relates the measured visibilities to the spherical harmonic coefficients of the sky signal. We use this to calculate window functions which relate the two-visibility correlations i.e. the correlation between the visibilities measured at two baselines and two frequencies, to different multipoles of the multi-frequency angular power spectrum $C_{\ell}\left(\nu_{1}, \nu_{2}\right)$. The formalism here is validated using simulations. We also present approximate closed form analytical expressions which can be used to calculate the window functions. Comparing the widely adopted flat sky approximation, we find that its predictions match those of our spherical harmonic formalism to within $16 \%$ across the entire OWFA baseline range. The match improves at large baselines where we have $<5 \%$ deviations.
\end{abstract}

Key words: Interferometric; cosmology: observations, diffuse radiation, largescale structure of Universe.

\footnotetext{
* E-mail: suman05@phy.iitkgp.ernet.in

† E-mail: somnath@phy.iitkgp.ernet.in 


\section{INTRODUCTION}

Intensity mapping with neutral Hydrogen Hi 21-cm radiation is a promising tool to study the large scale structures in the post-reionization Universe (Bharadwaj et al. 2001). The redshifted Hi 21-cm observations hold the potential of measuring the Baryon Acoustic Oscillation (BAO) that is embedded in the power spectrum of Hi 21-cm intensity fluctuations at all redshifts and the comoving scale of $\mathrm{BAO}$ can be used as a standard ruler to constrain the evolution of the equation of state for dark energy (Wyithe et al. 2008; Chang et al. 2008; Seo et al. 2010; Masui et al. 2010). Further, a measurement of just the Hi 21-cm power spectrum can also be used to constrain the cosmological parameters (Bharadwaj et al. 2009; Visbal et al. 2009). The higher order statistics such as the Hi 21-cm bispectrum holds the prospect of quantifying the non-Gaussianities in the Hi 21-cm signal (Ali et al. 2005; Hazra \& Sarkar 2012). Using the Hi signal in cross-correlation with the WiggleZ galaxy survey data, the Green Bank Telescope (GBT) has made the first detection of the Hi signal in emission at $z \approx 0.8$ (Chang et al. 2010).

A number of post-reionization experiments are either being planned or are ongoing at present. The Giant Meterwave Radio telescope (GMRT; Swarup et al. 1991) is sensitive to the cosmological Hi signal from a range of redshifts in the post-reionization era (Bharadwaj \& Pandey 2003; Bharadwaj \& Ali 2005). The upgraded GMRT (uGMRT; Gupta et al. 2017) is expected to have a larger bandwidth for which the prospects of a detection are investigated in Chatterjee et. al. 2018(in preparation). The Canadian Hydrogen Intensity Mapping Experiment (CHIME; Bandura et al. 2014) and the Hydrogen Intensity and Realtime Analysis eXperiment (HIRAX; Newburgh et al. 2016) aims to measure the BAO in the redshift range $0.8-2.5$. Future experiments, like Tianlai (Chen 2012, 2015) and SKA1-MID (Bull et al. 2015) also aim to measure the Hi 21-cm signal from the post-reionization era.

The Ooty Wide Field Array (OWFA; Subrahmanya et al. 2017a) is an upgraded version of the Ooty Radio Telescope (ORT, Swarup et al. 1971). The upgrade will result in two concurrently functioning modes named Phase I (PI) and Phase II (PII). The primary science goals of OWFA have been outlined in Subrahmanya et al. (2017b), and the measurement of the $\mathrm{z}=3.35$ post-reionization Hi 21-cm power spectrum is one of its major objectives (Ali \& Bharadwaj 2014). It has been predicted that a $5 \sigma$ detection of the amplitude of the Hi 21-cm power spectrum is possible with $\sim 150 \mathrm{hrs}$ of observation (Bharadwaj et al. 2015). Further, Sarkar et al. (2018) have predicted that a $\sim 5 \sigma$ measurement of the binned Hi 21- 
cm power spectrum is possible in the $k$-range $0.05 \mathrm{Mpc}^{-1} \leqslant k \leqslant 0.3 \mathrm{Mpc}^{-1}$ with $1,000 \mathrm{hrs}$ of observation.

The primary observable quantity for radio telescopes are the visibilities, which are the correlation of the measured voltages at each antenna. It is possible to directly estimate the redshifted Hi 21-cm power spectrum from the visibilities measured by a low frequency radio interferometric array (Bharadwaj \& Sethi 2001; Bharadwaj \& Ali 2005). Ali \& Bharadwaj (2014) presents theoretical predictions for the two-visibility correlations i.e. the correlation between the visibilities measured at two baselines and two frequencies, expected at OWFA considering both the HI 21-cm signal and also various foreground components. Chatterjee et al. (2017) and Marthi et al. (2017) have used numerical simulations to respectively predict the $21-\mathrm{cm}$ signal and the various foreground contributions to the two-visibility correlations expected at OWFA. Considering OWFA, Sarkar et al. (2018) presents an analytic technique to simulate the expected $21-\mathrm{cm}$ visibility signal. The common assumption of all the earlier works mentioned here is that the field of view (FoV) of the telescope is sufficiently small so that the observed sky can be assumed to be flat. In fact the flat sky approximation (FSA) is an underlying assumption in a large fraction of the works related to measuring the cosmological 21-cm power spectrum (e.g. Morales \& Hewitt 2004, Ali et al. 2015). Considering OWFA (both PI and PII), the FoV covers $1.8^{\circ}$ in the E-W direction while this is $4.8^{\circ}$ and $28.6^{\circ}$ in the N-S direction for PI and PII respectively. We expect the FSA to be a reasonably good approximation for PI, however for PII the large N-S extent of the FoV brings to question the validity of this assumption. It is desirable to consider the spherical nature of the sky in making predictions for PII.

Shaw et al. (2014) have introduced the "m-mode" formalism which incorporates the spherical nature of the sky. This essentially deals with drift scan observations, however their results can also be used to predict the signal for observations where the telescope tracks a fixed region of the sky. Liu et al. (2016) have introduced a spherical Fourier-Bessel technique for analysing the 21-cm power spectrum, that incorporates the spherical nature of the sky. Zhang et al. (2016a) and Ghosh et al. (2018) present sky map reconstruction methods based on the spherical harmonics ( $\mathrm{SH})$ transformation.

In this work, we develop a spherical sky formalism which is particularly suited for telescopes like OWFA, where the baselines are all coplanar with the antenna aperture. The formalism relates the visibilities to the SH coefficients of the sky signal through the "beam 


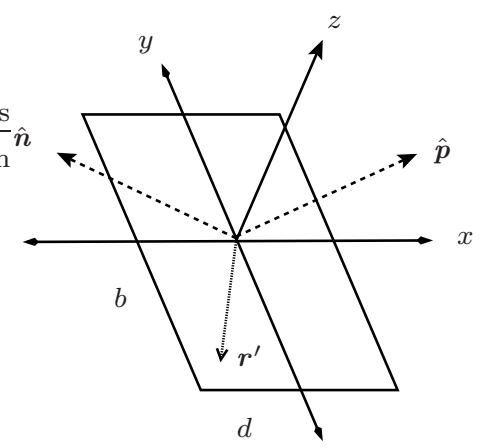

Figure 1. This shows the aperture of one of the OWFA antennas. The unit vector $\hat{p}$ is the pointing direction of the antenna beam, $\hat{n}$ points towards an arbitrary direction and $r^{\prime}$ refers to a displacement on the antenna aperture. The $x$ and $y$ axes are along the N-S and E-W directions respectively, while the $z$ axis, which is normal to the aperture, points towards the celestial Equator $(\delta=0)$.

transfer function". Section 2 of this paper presents a brief overview of OWFA, whereas the formalism is presented in Section 3. In addition to the numerical evaluation of the beam transfer function, we also use the Limber approximation (LA) to obtain an analytical expression for the same. In Section 4 we use the formalism to relate the two visibility correlations to the multi-frequency angular power spectrum (MAPS, Datta et al. 2007) which quantifies the statistics of the 21-cm signal. In Section 5 we validate our formalism using all-sky simulations. We study the differences between the FSA and the formalism of this paper in order to quantify how important the spherical nature of the sky is for the different phases of OWFA. The Results are presented in Section 6 and we present Summary and Conclusion in Section 7.

\section{OWFA}

The Ooty Radio Telescope (ORT) is a $530 \mathrm{~m}$ long (N-S) and $30 \mathrm{~m}$ wide (E-W) offset-parabolic cylinder, operating at a nominal frequency of $326.5 \mathrm{MHz}$. The telescope is equatorially mounted, i.e. the long axis of the reflecting cylinder is in the North-South direction, parallel to the Earth's rotation axis. The telescope can be mechanically steered along the East-West direction by a single rotation along the axis of the cylinder. The feed consists of 1056 NorthSouth dipoles of length $0.5 \lambda$ arranged nearly end to end along the focal line of the reflector. The telescope has a $530 \mathrm{~m} \times 30 \mathrm{~m}$ rectangular aperture.

Currently this telescope is being upgraded (Subrahmanya et al. 2017a) to operate as an interferometer the Ooty Wide Field Array (OWFA). The upgrade will result in two concurrent modes namely OWFA PI and PII respectively. In OWFA PI, the signals from $N_{d}=24$ adjacent dipoles will be combined to form a single antenna. Each antenna has a 

rectangular aperture of dimension $b \times d$, where $b=30 \mathrm{~m}$ and $d=11.5 \mathrm{~m}$ respectively. The array will have $N_{A}=40$ such antennas separated by $d=11.5 \mathrm{~m}$, arranged along the NorthSouth axis of the cylinder. In OWFA PII, the signals from $N_{d}=4$ adjacent dipoles will be combined to form a single antenna. Each antenna has a rectangular aperture of dimension $b \times d$, where $b=30 \mathrm{~m}$ and $d=1.92 \mathrm{~m}$ respectively. The array will have $N_{A}=264$ such antennas separated by $d=1.92 \mathrm{~m}$ arranged along the North-South axis of the cylinder.

Figure 1 shows a schematic diagram of the aperture for one of the OWFA antennas. For both PI and PII this is a rectangle of dimensions $b \times d$ with $b=30 \mathrm{~m}$, however as discussed earlier the dimension $d$ is different for the two modes. In both the modes, the digitised signals from $N_{d}$ successive dipoles are combined to form the antenna beam. This allows the pointing direction $\hat{\boldsymbol{p}}$ of the antenna beam pattern (Figure 1) to be steered electronically along the North-South direction by introducing phases. The antenna primary beam pattern $A(\Delta \hat{\boldsymbol{n}}, \nu)$ quantifies how the individual antenna responds to the signal from different directions $\hat{\boldsymbol{n}}$ on the sky (Figure 1 ), here $\Delta \hat{\boldsymbol{n}}=\hat{\boldsymbol{n}}-\hat{\boldsymbol{p}}$. It is possible to calculate $A(\Delta \hat{\boldsymbol{n}}, \nu)$ (e.g Chengalur et al. 2007) using,

$A(\Delta \hat{\boldsymbol{n}}, \nu)=\int d^{2} \boldsymbol{U}^{\prime} e^{2 \pi i \boldsymbol{U}^{\prime} \cdot \Delta \hat{\boldsymbol{n}}} \tilde{a}\left(\boldsymbol{U}^{\prime}, \nu\right)$

where, $\boldsymbol{U}^{\prime}=\boldsymbol{r}^{\prime} / \lambda$ refers to displacement $\boldsymbol{r}^{\prime}$ on the antenna aperture and the aperture power pattern $\tilde{a}\left(\boldsymbol{U}^{\prime}, \nu\right)$ is the auto-convolution of the electric field pattern at the antenna aperture (Figure 1). It is useful to note that the phase factor $e^{2 \pi i U^{\prime} \cdot \Delta \hat{n}}$ in eq. (1) is invariant under a mirror reflection with respect to the aperture plane. It follows that eq. (1) predicts a primary beam pattern which is exactly identical in the upper hemisphere $(U H)$ and lower hemisphere $(L H)$ of the sky. The telescope, however, only responds to the $U H$ and the $L H$ is not accessible to the telescope. Here we have exploited the fact that eq. (1) predicts an identical beam pattern in both the $U H$ and the $L H$ to simplify the mathematical analysis in subsequent parts of this paper.

For the purpose of the present analysis we make the simplifying assumption that the aperture is uniformly illuminated such that the electric field is uniform everywhere on the $b \times d$ rectangular aperture of the OWFA antenna. In this case the OWFA the aperture power pattern can be expressed as (Ali \& Bharadwaj 2014),

$\tilde{a}\left(\boldsymbol{U}^{\prime}, \nu\right)=\frac{\lambda^{2}}{b d} \Lambda\left(\frac{U_{x}^{\prime}}{d} \lambda\right) \Lambda\left(\frac{U_{y}^{\prime}}{b} \lambda\right)$ 


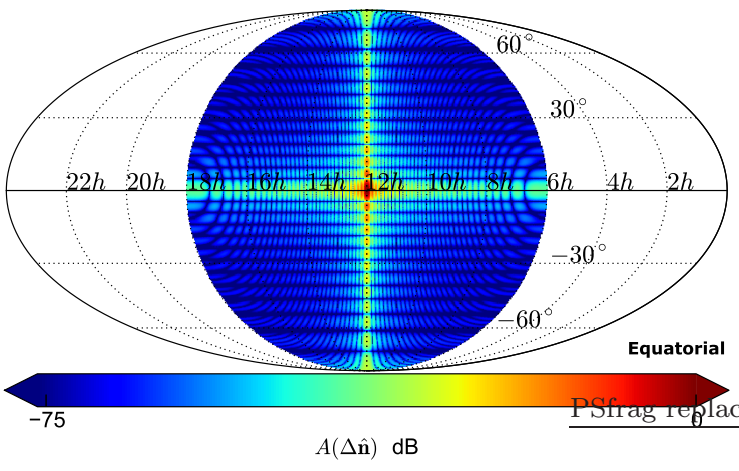

(a) Primary beam pattern for PI

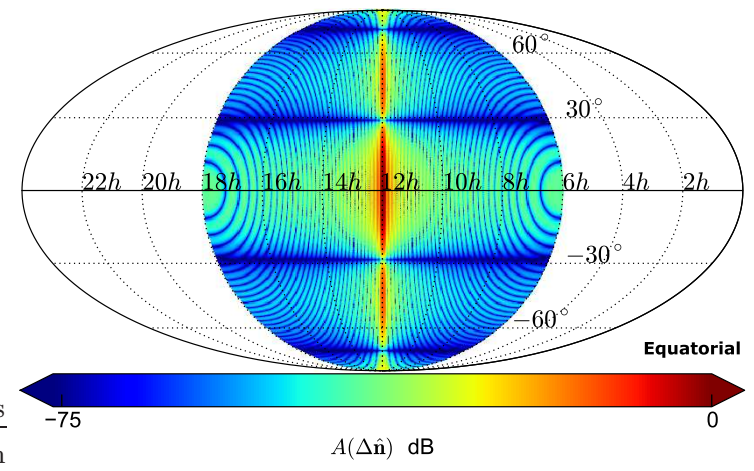

(b) Primary beam pattern for PII

Figure 2. The left and right panels show the primary beam pattern on the visible upper hemisphere for OWFA PI and PII respectively.

where $\boldsymbol{U}^{\prime}=\left(U_{x}^{\prime}, U_{y}^{\prime}\right), \Lambda(\xi)$ is the triangular function defined as, $\Lambda(\xi)=1-|\xi|$ for $|\xi|<1$ and $\Lambda(\xi)=0$ for $|\xi| \geqslant 1$ (see Figure (2a) of Ali \& Bharadwaj 2014).

We see that for OWFA, $\tilde{a}\left(\boldsymbol{U}^{\prime}, \nu\right)$ peaks at $\boldsymbol{U}^{\prime}=0$ and falls off as $\boldsymbol{U}^{\prime}$ is increased. Further this has compact support and $\tilde{a}\left(\boldsymbol{U}^{\prime}, \nu\right)=0$ if $\boldsymbol{U}^{\prime}$ exceeds the aperture dimensions. Note that these properties of $\tilde{a}\left(\boldsymbol{U}^{\prime}, \nu\right)$ are not particular to OWFA alone. In general, for any antenna, we expect $\tilde{a}\left(\boldsymbol{U}^{\prime}, \nu\right)$ to peak at $\boldsymbol{U}^{\prime}=0$, fall off as $\boldsymbol{U}^{\prime}$ is increased and to have compact support whereby $\tilde{a}\left(\boldsymbol{U}^{\prime}, \nu\right)=0$ if $\boldsymbol{U}^{\prime}$ exceeds the aperture dimensions.

Using equation (1) to calculate the OWFA primary beam pattern $A(\Delta \boldsymbol{n}, \nu)$, we get the product of two sinc-squared functions

$A(\Delta \boldsymbol{n}, \nu)=\operatorname{sinc}^{2}\left(\frac{\pi b \nu \Delta n_{y}}{c}\right) \operatorname{sinc}^{2}\left(\frac{\pi d \nu \Delta n_{x}}{c}\right)$

where $\Delta n_{y}$ and $\Delta n_{x}$ are respectively the $y$ and $x$ components of $\Delta \boldsymbol{n}$ (Marthi et al. 2017).

Figure 2 shows the primary beam pattern for OWFA PI and PII as a function of the celestial coordinates $(\alpha, \delta)$ on the visible upper hemisphere of the sky. Here the telescope beam is assumed to point towards $\hat{\boldsymbol{p}}=(\alpha, \delta)=(0,0)$. The OWFA beam is the diffraction pattern of a rectangular slit of dimension $b \times d$. The beam pattern is normalized to $A(\Delta \boldsymbol{n}, \nu)=1$ at the maxima which occurs when $\Delta \boldsymbol{n}=0$ (i.e. $\boldsymbol{n}=\hat{\boldsymbol{p}}$ ), and $A(\Delta \boldsymbol{n}, \nu)$ falls off as $\Delta \boldsymbol{n}$ is increased. The angular extent of the main lobe is determined by the first null which occurs at $\alpha_{0} \approx \lambda / b= \pm 1.8^{\circ}$ along $\alpha$ for both PI and PII, and at $\delta_{0} \approx \pm 4.8^{\circ}$ and $\pm 28.6^{\circ}$ along $\delta$ for PI and PII respectively. After the first nulls, there are side lobes in the OWFA primary beam pattern. The maxima of the $1^{\text {st }}$ and $2^{\text {nd }}$ side lobes are respectively $5 \%$ and $2 \%$ of the maxima of the main lobe.

The Fourier relation (eq. 1) implies that the primary beam pattern gets wider if the 


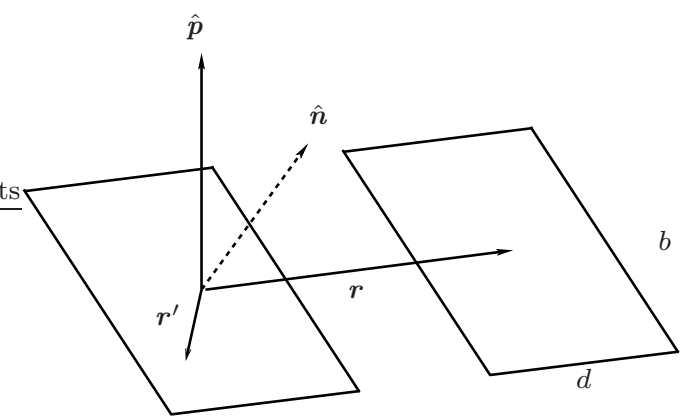

Figure 3. This shows a pair of antennas in a radio interferometer. Here $r$ is the antenna separation while $\hat{\boldsymbol{p}}, \hat{\boldsymbol{n}}$ and $\boldsymbol{r}^{\prime}$ are as defined in Figure 1.

antenna aperture dimensions are reduced. Here, we see that for PI the angular extent of the main lobe, which defines the antenna's FoV, is small and restricted within a few degrees on the sky. For this small region $(\theta \ll 1$ in radians) it is a reasonably good approximation to neglect the curvature of the sky, and it is adequate to carry out any further analysis for OWFA PI using the FSA (e.g. Marthi et al. 2017). For PII, however, we find that the FoV extends across $57.2^{\circ}$ in the N-S direction. It is not possible to ignore the curvature of the sky over such large angular extent, and it is necessary to consider the spherical sky for any further analysis for PII. Considering a radio-interferometer like OWFA, in the next Section we develop a formalism to analyze the measured visibilities in terms of a SH expansion which provide a natural basis for the signal on a spherical sky.

\section{VISIBILITIES AND BEAM TRANSFER FUNCTIONS}

The complex visibilities $\mathcal{V}(\boldsymbol{U}, \nu)$ are the quantities measured in a radio interferometric array. Here the baseline $\boldsymbol{U}$ refers to a pair of antennas separated by $\boldsymbol{r}$ (Figure 3), and $\boldsymbol{U}=\boldsymbol{r} / \lambda$ is the antenna separation in units of the observing wavelength. The baselines available at OWFA are

$\boldsymbol{U}_{n}=n \frac{d}{\lambda} \hat{\boldsymbol{i}} \quad\left(1 \leqslant n \leqslant N_{A}-1\right)$

where the values of $N_{A}$ and $d$ are different for PI and PII respectively, and the $(x, y, z)$ coordinate system is as shown in Figure 1.

We then have the visibility given by (Perley et al. 1989)

$\mathcal{V}(\boldsymbol{U}, \nu)=Q_{\nu} \int_{U H} d \Omega_{\hat{\boldsymbol{n}}} T(\hat{\boldsymbol{n}}, \nu) A(\Delta \boldsymbol{n}, \nu) e^{-2 \pi i \boldsymbol{U} \cdot \Delta \boldsymbol{n}}$,

where, $Q_{\nu}=2 k_{B} / \lambda^{2}$ is the conversion factor from brightness temperature to specific intensity in the Raleigh - Jeans limit, $T(\hat{\boldsymbol{n}}, \nu)$ is the brightness temperature distribution on the sky and $d \Omega_{\hat{n}}$ is the elemental solid angle in the direction $\hat{\boldsymbol{n}}$. For the $d \Omega_{\hat{\boldsymbol{n}}}$ integral, we use $U H, L H$ 
and $S$ to denote the upper hemisphere, the lower hemisphere and the entire celestial sphere respectively. Note that the integral here is over the visible upper hemisphere of the sky. The entire analysis in the rest of this sub-section is restricted to a single frequency, and in much of the subsequent text we do not show $\nu$ explicitly.

For a telescope with a sufficiently large aperture, the primary beam pattern $A(\Delta \hat{\boldsymbol{n}})$ falls off rapidly as $\Delta \boldsymbol{n}$ is increased. This restricts the FoV to a small region of the sky. Considering the small FoV, we can adopt the FSA whereby $\Delta \boldsymbol{n}=\boldsymbol{\theta}$ is a $2 \mathrm{D}$ vector on the plane of the sky with $d \Omega_{\hat{\boldsymbol{n}}}=d^{2} \boldsymbol{\theta}$. It is now natural to interpret $\mathcal{V}(\boldsymbol{U})$ (eq. 5) as the Fourier transform of the product $T(\boldsymbol{\theta}) A(\boldsymbol{\theta})$. Here, the visibility can be expressed as a convolution (Ali \& Bharadwaj 2014)

$$
\mathcal{V}(\boldsymbol{U})=Q \int d^{2} \boldsymbol{U}^{\prime} \tilde{a}\left(\boldsymbol{U}-\boldsymbol{U}^{\prime}\right) \tilde{T}\left(\boldsymbol{U}^{\prime}\right)
$$

where $\tilde{T}\left(\boldsymbol{U}^{\prime}\right)$ is the Fourier transform of the brightness temperature distribution on the sky. We may interpret $\mathcal{V}(\boldsymbol{U})$ as the weighted sum of different Fourier modes $\tilde{T}\left(\boldsymbol{U}^{\prime}\right)$ with the weights being given by the shifted aperture power pattern $\tilde{a}\left(\boldsymbol{U}-\boldsymbol{U}^{\prime}\right)$. The contribution peaks at $\boldsymbol{U}^{\prime}=\boldsymbol{U}$ where the value of $\tilde{a}\left(\boldsymbol{U}-\boldsymbol{U}^{\prime}\right)$ is maximum. This tells us that we may associate each visibility $\mathcal{V}(\boldsymbol{U})$ with a particular Fourier component $\tilde{T}(\boldsymbol{U})$ of the signal. To be more precise, the sum extends over a width $\left|\boldsymbol{U}-\boldsymbol{U}^{\prime}\right|$ which is of the order of the dimensions of the antenna aperture in units of the observing wavelength. As discussed earlier, $\tilde{a}\left(\boldsymbol{U}-\boldsymbol{U}^{\prime}\right)$ is zero beyond the extent of the antenna aperture.

The FSA breaks down for the antennas with a small aperture which have a large FoV. Here, one has to consider the spherical nature of the sky. The $\mathrm{SH}$ function $Y_{\ell}^{m}(\hat{\boldsymbol{n}})$ provide a natural basis for the signal on a spherical sky, and in the SH expansion we have

$T(\hat{\boldsymbol{n}}, \nu)=\sum_{\ell, m} a_{\ell}^{m}(\nu) Y_{\ell}^{m}(\hat{\boldsymbol{n}})$,

where $a_{\ell}^{m}$ are the SH coefficients of the brightness temperature distribution.

As discussed earlier by Shaw et al. (2014, 2015) (and also by Zheng et al. 2014; Zhang et al. 2016a,b; Liu et al. 2016), the visibilities can be expressed in terms of the SH coefficients $a_{\ell}^{m}$ as,

$\mathcal{V}(\boldsymbol{U})=\sum_{\ell, m} a_{\ell}^{m} B_{\ell}^{m}(\boldsymbol{U})$

The visibility expressed in eq. (8) is a weighted sum of the SH coefficients $a_{\ell}^{m}$ with the weights being given by the beam transfer function $B_{\ell}^{m}(\boldsymbol{U})$. Note that this is in exact analogy with 
eq. (6) with the difference that in the FSA we use Fourier modes $\tilde{T}(\boldsymbol{U})$ instead of the SH coefficients $a_{\ell}^{m}$, and we have the shifted aperture power pattern $\tilde{a}\left(\boldsymbol{U}-\boldsymbol{U}^{\prime}\right)$ as the weights instead of $B_{\ell}^{m}(\boldsymbol{U})$. This analogy between eqs. (8) and (6) leads to a picture where we interpret $B_{\ell}^{m}(\boldsymbol{U})$ as the spherical sky generalization of the shifted aperture power pattern $\tilde{a}\left(\boldsymbol{U}-\boldsymbol{U}^{\prime}\right)$.

The beam transfer function $B_{\ell}^{m}(\boldsymbol{U})$ which quantifies how a visibility $\mathcal{V}(\boldsymbol{U})$ responds to a particular SH coefficient $a_{\ell}^{m}$ can be calculated (Shaw et al. 2014) using

$B_{\ell}^{m}(\boldsymbol{U})=Q \int_{U H} d \Omega_{\hat{\boldsymbol{n}}} Y_{\ell}^{m}(\hat{\boldsymbol{n}}) A(\Delta \hat{\boldsymbol{n}}) e^{-2 \pi i \boldsymbol{U} \cdot \Delta \hat{\boldsymbol{n}}}$.

The integrand here contains two highly oscillatory functions $\left(Y_{\ell}^{m}(\hat{\boldsymbol{n}})\right.$ and $\left.e^{-2 \pi i \boldsymbol{U} \cdot \Delta \hat{\boldsymbol{n}}}\right)$, and the integral extends over the visible upper hemisphere of the sky. It is computationally expensive and cumbersome to evaluate such integrals, particularly for large values of $\ell$ and $\boldsymbol{U}$. The major contribution to the integral comes from the main lobe of the primary beam pattern, and one may consider using this to restrict the integral. However the main lobe itself extends over a large region of the sky for telescopes with a small aperture. Further, the sidelobes also contribute to the integral, and it is necessary to include the whole hemisphere to take this into account. Finally, we note that eq. (10) provides very little insight into the behaviour of $B_{\ell}^{m}(\boldsymbol{U})$ i.e. at which $\ell, m$ values we have the maximum contribution for a particular baseline $\boldsymbol{U}$.

In this paper we develop a different formalism for calculating the beam transfer function $B_{\ell}^{m}(\boldsymbol{U})$. As noted earlier, it is natural to interpret $B_{\ell}^{m}(\boldsymbol{U}, \nu)$ as the spherical sky counterpart of the shifted aperture power pattern $\tilde{a}\left(\boldsymbol{U}-\boldsymbol{U}^{\prime}\right)$, and in this paper we express $B_{\ell}^{m}(\boldsymbol{U})$ as an integral of the aperture power pattern $\tilde{a}\left(\boldsymbol{U}-\boldsymbol{U}^{\prime}\right)$. The $d \Omega_{\hat{n}}$ integrals in eqs. (5) and (10) are limited to the visible upper hemisphere $(U H)$. The SH functions cease to be orthonormal when the domain is restricted to a hemisphere. The analysis is considerably simplified if we assume that the primary beam pattern and also the sky signal are both replicated on the lower hemisphere $(L H)$ by a reflection with respect to the plane of the antenna aperture (the $x y$ plane in Figure 1 ). The domain of the $d \Omega_{\hat{n}}$ integrals are now extended to the entire sphere $(S)$. In this situation, the upper and lower hemispheres both make an equal contribution to the visibility signal. We account for this by introducing a factor of $1 / 2$ in the beam transfer function which is now defined as

$B_{\ell}^{m}(\boldsymbol{U})=\frac{Q}{2} \int_{S} d \Omega_{\hat{\boldsymbol{n}}} Y_{\ell}^{m}(\hat{\boldsymbol{n}}) A(\Delta \hat{\boldsymbol{n}}) e^{-2 \pi i \boldsymbol{U} \cdot \Delta \hat{\boldsymbol{n}}}$.

The fact that $Y_{\ell}^{m}(\hat{\boldsymbol{n}}) \rightarrow(-1)^{\ell+m} Y_{\ell}^{m}(\hat{\boldsymbol{n}})$ under a mirror reflection with respect to the $x y$ MNRAS 000, 1-?? (2018) 
plane implies that $B_{\ell}^{m}(\boldsymbol{U})=0$ when $\ell+m$ is odd, and $B_{\ell}^{m}(\boldsymbol{U})$ is non-zero only when $\ell+m$ is even.

It is necessary to note that $a_{\ell}^{m}$ in eq. (8) now refers to the SH expansion of the replicated brightness temperature signal $T(\hat{\boldsymbol{n}})$ i.e. the signal in the $L H$ is a mirror reflection of the signal in the visible $U H$, the reflection here is with respect to the plane of the antenna aperture.

We proceed by expressing $A(\Delta \hat{\boldsymbol{n}})$ in eq. (10) in terms of the aperture power pattern $\tilde{a}\left(\boldsymbol{U}-\boldsymbol{U}^{\prime}\right)$ using eq. (1) which gives us

$B_{\ell}^{m}(\boldsymbol{U})=\frac{Q}{2} \int_{S} d \Omega_{\hat{n}} Y_{\ell}^{m}(\hat{\boldsymbol{n}}) e^{-2 \pi i \boldsymbol{U} \cdot \Delta \hat{n}} \int d^{2} \boldsymbol{U}^{\prime} e^{2 \pi i \boldsymbol{U}^{\prime} \cdot \Delta \hat{n}} \tilde{a}\left(\boldsymbol{U}-\boldsymbol{U}^{\prime}\right)$.

Note that the $d \Omega_{\hat{n}}$ integral in eq. (11) is over the entire sphere.

The subsequent analysis is considerably simplified if we assume that the baselines $\boldsymbol{U}$ are coplanar with the antenna aperture. This assumption is true for OWFA (Figure 3). We also expect this to be a good approximation for any compact array of fixed antennas which point vertically overhead. Under this assumption, $\boldsymbol{U}$ and $\boldsymbol{U}^{\prime}$ in eq. (11) are co-planer vectors, and we can write

$B_{\ell}^{m}(\boldsymbol{U})=\frac{Q}{2} \int d^{2} \boldsymbol{U}^{\prime} \tilde{a}\left(\boldsymbol{U}-\boldsymbol{U}^{\prime}\right)\left[\int_{S} d \Omega_{\hat{\boldsymbol{n}}} Y_{\ell}^{m}(\hat{\boldsymbol{n}}) e^{2 \pi i \boldsymbol{U}^{\prime} \cdot \Delta \hat{\boldsymbol{n}}}\right]$.

We use the identity

$e^{2 \pi i \boldsymbol{U}^{\prime} \cdot \hat{\boldsymbol{n}}}=4 \pi \sum_{\ell, m} i^{\ell} Y_{\ell}^{m}\left(\hat{\boldsymbol{U}}^{\prime}\right) Y_{\ell}^{m *}(\hat{\boldsymbol{n}}) j_{\ell}\left(2 \pi\left|\boldsymbol{U}^{\prime}\right|\right)$

to evaluate the integral in the square brackets of eq. (12), here $j_{\ell}\left(2 \pi\left|\boldsymbol{U}^{\prime}\right|\right)$ is the $\ell^{\text {th }}$ order spherical Bessel function of first kind. We finally obtain

$B_{\ell}^{m}(\boldsymbol{U})=2 \pi i^{\ell} Q \int d^{2} \boldsymbol{U}^{\prime} \tilde{a}\left(\boldsymbol{U}-\boldsymbol{U}^{\prime}\right) Y_{\ell}^{m}\left(\hat{\boldsymbol{U}}^{\prime}\right) j_{\ell}\left(2 \pi\left|\boldsymbol{U}^{\prime}\right|\right) e^{-2 \pi i \boldsymbol{U}^{\prime} \cdot \hat{\boldsymbol{p}}}$,

which we use to compute the beam transfer function $B_{\ell}^{m}(\boldsymbol{U})$.

The integrand here contains three highly oscillatory functions $\left(Y_{\ell}^{m}\left(\hat{\boldsymbol{U}}^{\prime}\right), j_{\ell}\left(2 \pi\left|\boldsymbol{U}^{\prime}\right|\right)\right.$ and $\left.e^{-2 \pi i \boldsymbol{U}^{\prime} \cdot \hat{\boldsymbol{p}}}\right)$, however in contrast to eq. (10), the domain of the integral is restricted by $\tilde{a}(\boldsymbol{U}-$ $\left.\boldsymbol{U}^{\prime}\right)$ which has compact support. As mentioned earlier, $\tilde{a}\left(\boldsymbol{U}-\boldsymbol{U}^{\prime}\right)$ is zero if $\boldsymbol{U}-\boldsymbol{U}^{\prime}$ exceeds the aperture dimensions in units of the observing wavelength. This significantly reduces the computation for calculating $B_{\ell}^{m}(\boldsymbol{U})$, particularly for small apertures where the primary beam pattern covers a large region of the sky.

The subsequent analysis is restricted to the situation where the pointing direction $\hat{\boldsymbol{p}}$ of the antenna beam pattern is perpendicular to the antenna aperture so that we have $e^{-2 \pi i \boldsymbol{U}^{\prime} \cdot \hat{\boldsymbol{p}}}=1$ for the phase factor in eq. (14). We adopt the coordinate system shown in Figure 1 with the 


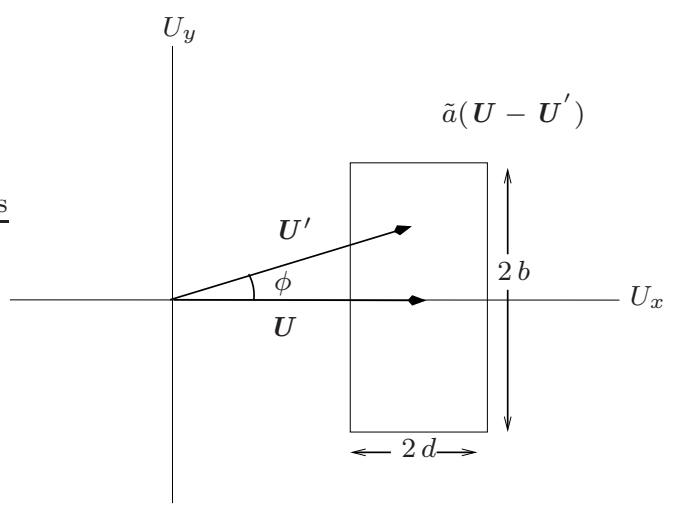

Figure 4. This shows the domain of integration in eq. (15). Here $U$ corresponds to the baseline, $U^{\prime}$ and $\phi$ can vary with in the aperture of the antenna (shown by the rectangle). $\phi$ becomes significantly small for large baseline $|\boldsymbol{U}|>>d / \lambda$. The $U_{x}-U_{y}$ plane here corresponds to the $\theta=\pi / 2$ for the previously adopted spherical polar coordinate system.

baseline $\boldsymbol{U}$ (Figure 3) aligned with $x$ axis. Expressing eq. (14) in spherical polar coordinates, the vector $\boldsymbol{U}^{\prime}$ is restricted to the $\theta=\frac{\pi}{2}$ plane and we then have $Y_{\ell}^{m}\left(\hat{\boldsymbol{U}}^{\prime}\right)=Y_{\ell}^{m}(\pi / 2,0) e^{i m \phi}$. So the beam transfer function $B_{\ell}^{m}(\boldsymbol{U})$ can be written as

$B_{\ell}^{m}(\boldsymbol{U})=2 \pi i^{\ell} Y_{\ell}^{m}\left(\frac{\pi}{2}, 0\right) Q \int d U^{\prime} U^{\prime} j_{\ell}\left(2 \pi U^{\prime}\right) \int d \phi \tilde{a}\left(U-U^{\prime} \cos \phi, U^{\prime} \sin \phi\right) e^{i m \phi}$,

where the domain of integration is shown in Figure 4. The integral here contains two highly oscillatory functions $j_{\ell}\left(2 \pi U^{\prime}\right)$ and $e^{i m \phi}$.

Considering the complex conjugate $\left[B_{\ell}^{m}(\boldsymbol{U})\right]^{*}$, the fact that $Y_{l}^{m *}(\theta, \phi)=(-1)^{m} Y_{l}^{-m}(\theta, \phi)$ implies that $\left[B_{\ell}^{m}(\boldsymbol{U})\right]^{*}=B_{\ell}^{-m}(\boldsymbol{U})$. If we further assume that the aperture power pattern is symmetric with respect to $U_{y}$ i.e. $\tilde{a}\left(U_{x},-U_{y}\right)=\tilde{a}\left(U_{x}, U_{y}\right)$ (which is true for OWFA) we have $\left[B_{\ell}^{m}(\boldsymbol{U})\right]^{*}=(-1)^{\ell} B_{\ell}^{m}(\boldsymbol{U})$. This implies that for even $\ell$ we have the non-zero elements $B_{\ell}^{0}(\boldsymbol{U}), B_{\ell}^{2}(\boldsymbol{U})=B_{\ell}^{-2}(\boldsymbol{U}), B_{\ell}^{4}(\boldsymbol{U})=B_{\ell}^{-4}(\boldsymbol{U}), \ldots, B_{\ell}^{\ell}(\boldsymbol{U})=B_{\ell}^{-\ell}(\boldsymbol{U})$ which are all real. For odd $\ell$ we have the non-zero elements $B_{\ell}^{1}(\boldsymbol{U})=-B_{\ell}^{-1}(\boldsymbol{U}), B_{\ell}^{3}(\boldsymbol{U})=-B_{\ell}^{-3}(\boldsymbol{U}), \ldots, B_{\ell}^{\ell-1}(\boldsymbol{U})=$ $-B_{\ell}^{-\ell+1}(\boldsymbol{U})$ which are all imaginary. The other elements of $B_{\ell}^{m}(\boldsymbol{U})$ are all zero.

It is possible to obtain further insight into the behaviour of $B_{\ell}^{m}(\boldsymbol{U})$ if we adopt the LA (Limber 1954)

$j_{\ell}\left(2 \pi U^{\prime}\right) \approx \sqrt{\frac{\pi}{2 \ell+1}} \delta_{D}\left(\ell+1 / 2-2 \pi U^{\prime}\right)$.

which holds for large $\ell$. Here we also assume that the baseline is large compared to the antenna aperture dimensions $U \gg b / \lambda$ so that the $\phi$ range subtended by the aperture (Figure 4) is small whereby $\cos \phi \approx 1$ and $\sin \phi \approx \phi$. We then have

$B_{\ell}^{m}(\boldsymbol{U})=i^{\ell} \sqrt{\frac{2 \ell+1}{16 \pi}} Y_{\ell}^{m}\left(\frac{\pi}{2}, 0\right) Q \int d \phi \tilde{a}\left(U-\frac{2 \ell+1}{4 \pi}, \frac{2 \ell+1}{4 \pi} \phi\right) e^{i m \phi}$.

For OWFA we can decompose the primary beam pattern (eq. 3) as $A(\Delta \hat{\boldsymbol{n}})=A_{x}\left(\Delta n_{x}\right) A_{y}\left(\Delta n_{y}\right)$ and the aperture power pattern (eq. 2) as $\tilde{a}\left(\boldsymbol{U}^{\prime}\right)=\tilde{a}_{x}\left(U_{x}^{\prime}\right) \tilde{a}_{y}\left(U_{y}^{\prime}\right)$, where $A_{x}\left(\Delta n_{x}\right)$ and 


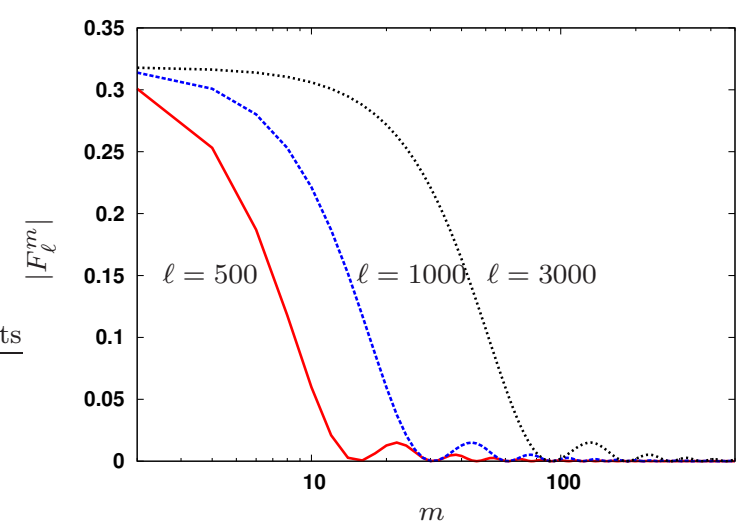

Figure 5. This shows the variation of $\left|F_{\ell}^{m}\right|$ as a function of $m$ for the fixed $\ell$ values 500 (solid line), 1000(dased line) and 3000(dotted line).

$A_{y}\left(\Delta n_{y}\right)$ are respectively the Fourier transforms (eq. 1) of $\tilde{a}_{x}\left(U_{x}^{\prime}\right)$ and $\tilde{a}_{y}\left(U_{y}^{\prime}\right)$. It is then possible to analytically evaluate the $\phi$ integral in eq. (17) and we have

$B_{\ell}^{m}(\boldsymbol{U})=i^{\ell} Q \sqrt{\frac{\pi}{2 \ell+1}} Y_{\ell}^{m}\left(\frac{\pi}{2}, 0\right) \tilde{a}_{x}\left(\boldsymbol{U}-\frac{2 \ell+1}{4 \pi}\right) A_{y}\left(\frac{m}{2 \ell+1}\right)$.

where $\tilde{a}_{x}\left(U_{x}^{\prime}\right)=(\lambda / d) \Lambda\left(U_{x}^{\prime} \lambda / d\right)$ and $A_{y}\left(\Delta n_{y}\right)=\operatorname{sinc}^{2}\left(\pi b \Delta n_{y} / \lambda\right)$ for OWFA.

It is interesting to note that, in contrast to eq. (10), the alternate expression (eq. 14) obtained here provides useful insight into the behaviour of the beam transfer function $B_{\ell}^{m}(\boldsymbol{U})$. We see that the integrand in eq. (14) is the product of $\tilde{a}\left(\boldsymbol{U}-\boldsymbol{U}^{\prime}\right)$, which is peaked at $\boldsymbol{U}=\boldsymbol{U}^{\prime}$, and $j_{\ell}\left(2 \pi\left|\boldsymbol{U}^{\prime}\right|\right)$, which is peaked at $\ell=2 \pi\left|\boldsymbol{U}^{\prime}\right|$. Based on this we can infer that $B_{\ell}^{m}(\boldsymbol{U})$ has a maximum value when $\ell=2 \pi|\boldsymbol{U}|$, and the value of $B_{\ell}^{m}(\boldsymbol{U})$ falls off as the difference between $\ell$ and $2 \pi|\boldsymbol{U}|$ is increased. In other words, the visibility $\mathcal{V}(\boldsymbol{U}, \nu)$ receives maximum contribution from the SH coefficients $a_{\ell}^{m}$ with $\ell=2 \pi|\boldsymbol{U}|$. The spread $\Delta \ell$ around this $\ell$ value depends on the aperture power pattern with $\Delta \ell \sim 2 \pi D / \lambda$ where $D$ represents the aperture size, i.e. we have a smaller spread in $\ell$ if the aperture is small as compared to a large aperture. This behaviour is explicit in the approximate analytical expression (eq. 18) for $B_{\ell}^{m}(\boldsymbol{U})$.

Eq. (18) provides further insight in the behaviour of $B_{\ell}^{m}(\boldsymbol{U})$. For a fixed $\ell$, it is possible to analyze the $m$ dependence of $B_{\ell}^{m}(\boldsymbol{U})$ by considering the function $F_{\ell}^{m}=Y_{\ell}^{m}\left(\frac{\pi}{2}, 0\right) A_{y}\left(\frac{m}{2 \ell+1}\right)$ which appears in eq. (18). Figure 5 shows the variation of $\left|F_{\ell}^{m}\right|$ as a function of $m$ for fixed $\ell$ values (mentioned in the figure). We find that the $m$ dependence of $\left|F_{\ell}^{m}\right|$ is very similar to $\operatorname{sinc}^{2}(\pi b m /(\lambda(2 \ell+1)))$. We expect the amplitude of $B_{\ell}^{m}(\boldsymbol{U})$ to be maximum for $m=0$ and decrease with increasing $m$.

Figure 6 shows the OWFA beam transfer function $B_{\ell}^{m}\left(\boldsymbol{U}_{a}\right)$ as a function of $\ell$ for a few fixed values of $\boldsymbol{U}_{a}$ with the $a$ and $m$ values as mentioned in the figure. The solid and dashed 

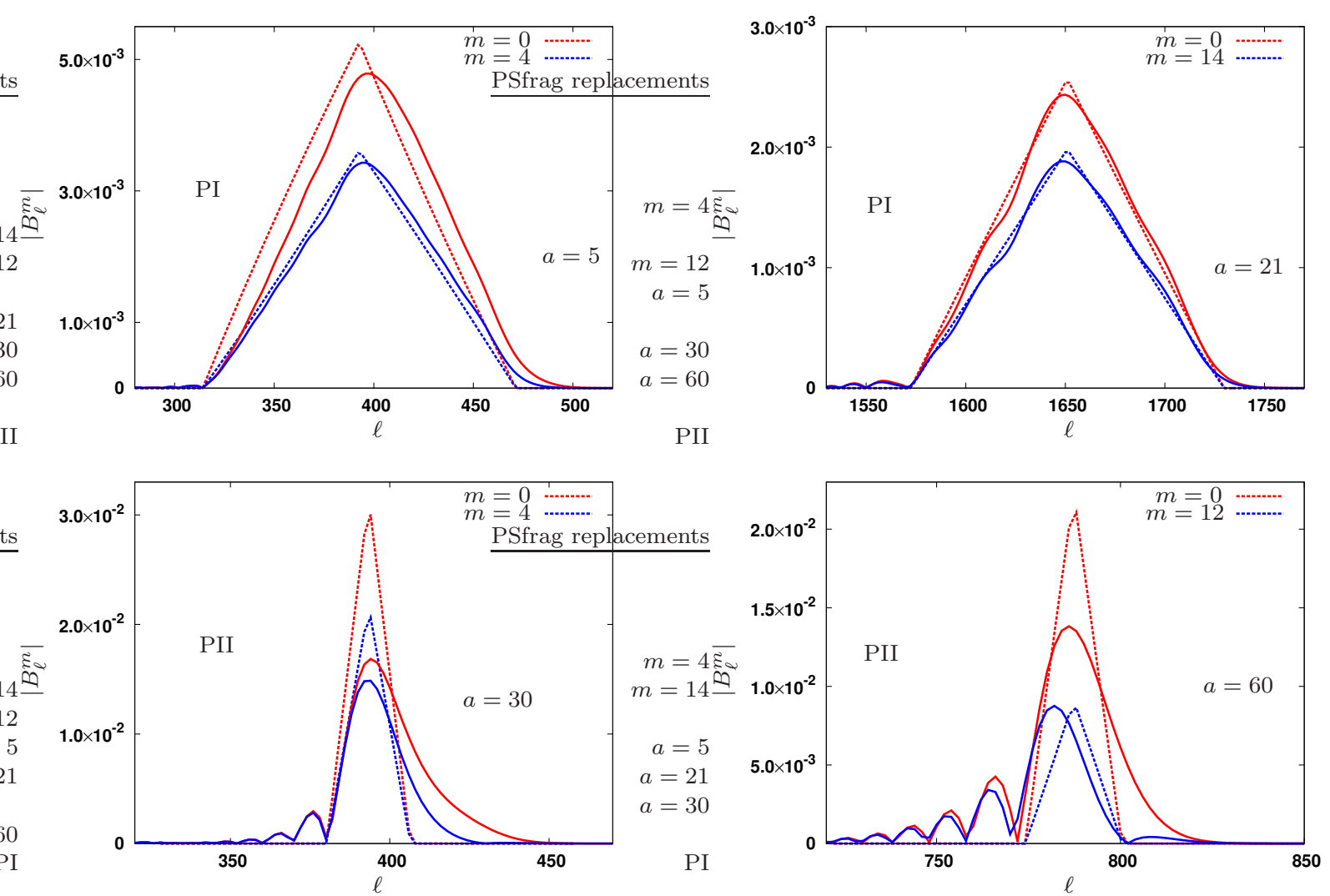

Figure 6. This shows the beam transfer function $B_{\ell}^{m}\left(\boldsymbol{U}_{a}\right)$ as a function of $\ell$ for fixed values of $\boldsymbol{U}_{a}$ with the $a$ and $m$ values as mentioned in the figure. The top and bottom rows show results for OWFA PI and PII respectively. The solid and dashed lines represent $B_{\ell}^{m}\left(\boldsymbol{U}_{a}\right)$ computed using eq. (14) and the LA (eq. 18) respectively.

lines correspond to $B_{\ell}^{m}\left(\boldsymbol{U}_{a}\right)$ computed using eq. (14) and the LA (eq. 18) respectively. The top row shows the results for OWFA PI where we see that $\left|B_{\ell}^{m}\left(\boldsymbol{U}_{a}\right)\right|$ peaks at $\ell_{a}=395$ and 1650 for $a=5$ and 21 respectively. This is consistent with $\ell_{a} \approx 2 \pi a d / \lambda$ where $d=11.5 \mathrm{~m}$ and $2 \pi d / \lambda \approx 79$. We also see that, as expected, for all values of $a$ the beam transfer function extends over a nearly fixed interval of $\Delta \ell= \pm 79$ around the peak value. We find that LA works very well for the larger baseline whereas it is in reasonable agreement for the smaller baseline. The bottom row of Figure 6 shows the results for OWFA PII where we see that $\left|B_{\ell}^{m}\left(\boldsymbol{U}_{a}\right)\right|$ peaks at $\ell_{a}=390$ and 782 for $a=30$ and 60 respectively, which is consistent with $\ell_{a} \approx 2 \pi a d / \lambda$ where $d=1.92 \mathrm{~m}$ and $2 \pi d / \lambda \approx 13$. However, unlike PI, here we see that $\left|B_{\ell}^{m}\left(\boldsymbol{U}_{a}\right)\right|$ shows several secondary peaks (oscillations) after the first zero to the left of $\ell_{a}$ and it shows a tail which extends to $\Delta \ell \approx 40$ to the right of $\ell_{a}$. These features are not present in the $B_{\ell}^{m}\left(\boldsymbol{U}_{a}\right)$ computed using LA and we find that the LA does not work well even for the large baselines (e.g. $a=60)$. The oscillations here are a manifestation of the oscillations in the Bessel function $j_{\ell}\left(2 \pi U^{\prime}\right)$ in eq. (15). For PI the aperture power pattern $\tilde{a}\left(\boldsymbol{U}-\boldsymbol{U}^{\prime}\right)$ is wider than the oscillation period of $j_{\ell}\left(2 \pi U^{\prime}\right)$ and these oscillations are averaged out in 
eq. (15). However, PII has a smaller aperture and consequently these oscillations persist in $B_{\ell}^{m}\left(\boldsymbol{U}_{a}\right)$.

\section{VISIBILITY CORRELATION AND ANGULAR POWER SPECTRUM}

The interest here is in a statistical detection of the redshifted cosmological 21-cm signal using radio-interferometric observations. In this section we consider a more general situation where we wish to detect an arbitrary statistical sky signal using radio-interferometric observations. We assume that the brightness temperature distribution $T(\hat{\boldsymbol{n}}, \nu)$ on the sky is the outcome of a statistically homogeneous and isotropic random process. Note that, this assumption requires $T(\hat{\boldsymbol{n}}, \nu)$ to be defined on the entire celestial sphere $S$. The two point statistics of this signal can be completely quantified using the multi-frequency angular power spectrum (MAPS; Datta et al. 2007) which jointly characterises the angular and the frequency dependence of this sky signal. The MAPS of the brightness temperature distribution at two different frequencies $\nu_{i}$ and $\nu_{j}$ is defined through

$\mathcal{C}_{\ell}\left(\nu_{i}, \nu_{j}\right)=\left\langle a_{\ell}^{m}\left(\nu_{i}\right) a_{\ell}^{m *}\left(\nu_{j}\right)\right\rangle$.

The measured visibilities $\mathcal{V}(\boldsymbol{U}, \nu)$ arising from this random signal are also random. We use two-visibility correlations (Bharadwaj \& Sethi 2001) to observationally quantify the statistical properties of the input sky signal. The two-visibility correlation

$V_{2}\left(\boldsymbol{U}_{a}, \nu_{i} ; \boldsymbol{U}_{b}, \nu_{j}\right) \equiv\left\langle\mathcal{V}\left(\boldsymbol{U}_{a}, \nu_{i}\right) \mathcal{V}^{*}\left(\boldsymbol{U}_{b}, \nu_{j}\right)\right\rangle$

refers to the correlation in the visibilities measured at two baselines $\boldsymbol{U}_{a}$ and $\boldsymbol{U}_{b}$, and frequencies $\nu_{i}$ and $\nu_{j}$. The angular brackets here denote an ensemble average over different random realization of the sky signal. The two point statistics, or equivalently the power spectrum (MAPS), of the sky signal is contained in this two-visibility correlation.

The calculations are fairly simplified in the FSA (Ali \& Bharadwaj 2014) where we have

$$
V_{2}\left(\boldsymbol{U}_{a}, \nu_{i} ; \boldsymbol{U}_{b}, \nu_{j}\right)=\left(\frac{\partial B}{\partial T}\right)^{2} \int d^{2} \boldsymbol{U}^{\prime} \tilde{a}\left(\boldsymbol{U}_{a}-\boldsymbol{U}^{\prime}, \nu_{i}\right) \tilde{a}\left(\boldsymbol{U}_{b}-\boldsymbol{U}^{\prime}, \nu_{j}\right) \mathcal{C}_{2 \pi U^{\prime}}\left(\nu_{i}, \nu_{j}\right) \text {, }
$$

which relates the visibility correlation to MAPS $\mathcal{C}_{2 \pi U}\left(\nu_{i}, \nu_{j}\right)$. This can be obtained by using eq. (6) in eq. (20), and finally utlizing the fact (Datta et al. 2007) that

$\left\langle\tilde{T}\left(\boldsymbol{U}, \nu_{i}\right) \tilde{T}\left(\boldsymbol{U}^{\prime}, \nu_{j}\right)\right\rangle=\delta_{D}^{2}\left(\boldsymbol{U}-\boldsymbol{U}^{\prime}\right) \mathcal{C}_{2 \pi U}\left(\nu_{i}, \nu_{j}\right)$

where $\delta_{D}^{2}\left(\boldsymbol{U}-\boldsymbol{U}^{\prime}\right)$ is the 2D Dirac delta function. This expression for the visibility correlation (eq. 21) is adequate for telescopes with small FoV (e.g. OWFA PI).

It is necessary to consider the SH formalism (eq. 8) for telescopes with a large FoV (e.g. 
OWFA PII). To obtain an expression for the visibility correlation in the SH formalism, we revisit the visibilities as discussed earlier (in eq. 5),

$\mathcal{V}(\boldsymbol{U})=Q \int_{U H} d \Omega_{\hat{\boldsymbol{n}}} T(\hat{\boldsymbol{n}}) A(\Delta \boldsymbol{n}) e^{-2 \pi i \boldsymbol{U} \cdot \Delta \boldsymbol{n}}$,

where the $d \Omega_{\hat{n}}$ integral is over the visible upper hemisphere $(U H)$. For our convenience, we introduce the visibility

$\mathcal{V}^{\prime}(\boldsymbol{U})=Q \int_{L H} d \Omega_{\hat{\boldsymbol{n}}} T(\hat{\boldsymbol{n}}) A(\Delta \boldsymbol{n}) e^{-2 \pi i \boldsymbol{U} \cdot \Delta n}$,

where the $d \Omega_{\hat{n}}$ integral is over the lower hemisphere $(L H)$ of the sky. Here we can safely assume that the major contributions of the sky signal to $\mathcal{V}(\boldsymbol{U})$ and $\mathcal{V}^{\prime}(\boldsymbol{U})$ are statistically uncorrelated which implies that the $\mathcal{V}(\boldsymbol{U})$ and $\mathcal{V}^{\prime}(\boldsymbol{U})$ are statistically uncorrelated $i$. $e$.

$\left\langle\left(\mathcal{V}(\boldsymbol{U})+\mathcal{V}^{\prime}(\boldsymbol{U})\right)\left(\mathcal{V}(\boldsymbol{U})+\mathcal{V}^{\prime}(\boldsymbol{U})\right)^{*}\right\rangle=\left\langle|\mathcal{V}(\boldsymbol{U})|^{2}\right\rangle+\left\langle\left|\mathcal{V}^{\prime}(\boldsymbol{U})\right|^{2}\right\rangle$

We also have,

$\left\langle|\mathcal{V}(\boldsymbol{U})|^{2}\right\rangle=\left\langle\left|\mathcal{V}^{\prime}(\boldsymbol{U})\right|^{2}\right\rangle$

Following the formalism discussed in the previous section, the total visibility can be expanded as,

$\mathcal{V}(\boldsymbol{U})+\mathcal{V}^{\prime}(\boldsymbol{U})=\sum_{\ell, m} b_{\ell}^{m} B_{\ell}^{m}(\boldsymbol{U})$

Here $b_{\ell}^{m}$ are the SH coefficients of the sky signal $T(\hat{\boldsymbol{n}})+T_{R}(\hat{\boldsymbol{n}})$, where $T_{R}(\hat{\boldsymbol{n}})$ is the $T(\hat{\boldsymbol{n}})$ replicated on the whole sphere $(S)$ after a reflection with respect to the aperture plane of the antenna. The statistical properties of $b_{\ell}^{m}$ can be quantified using the MAPS of the brightness temperature distribution $T(\hat{\boldsymbol{n}})$ (discussed in eq. 19) as,

$\left\langle b_{\ell}^{m}\left(\nu_{i}\right) b_{\ell^{\prime}}^{m^{\prime} *}\left(\nu_{j}\right)\right\rangle=4 \mathcal{C}_{\ell}\left(\nu_{i}, \nu_{j}\right) \delta_{\ell \ell^{\prime}} \delta_{m m^{\prime}}$.

We then obtain the visibility correlation to be

$\left\langle\mathcal{V}\left(\boldsymbol{U}_{a}, \nu_{i}\right) \mathcal{V}^{*}\left(\boldsymbol{U}_{b}, \nu_{j}\right)\right\rangle=\sum_{\ell} \mathcal{C}_{\ell}\left(\nu_{i}, \nu_{j}\right) \mathcal{W}_{\ell}\left(\boldsymbol{U}_{a}, \nu_{i} ; \boldsymbol{U}_{b}, \nu_{j}\right)$

where $\mathcal{W}_{\ell}$ is a window function which is defined as

$\mathcal{W}_{\ell}\left(\boldsymbol{U}_{a}, \nu_{i} ; \boldsymbol{U}_{b}, \nu_{j}\right)=2 \sum_{m} B_{\ell}^{m}\left(\boldsymbol{U}_{a}, \nu_{i}\right) B_{\ell}^{m *}\left(\boldsymbol{U}_{b}, \nu_{j}\right)$

Eqs. (21) and (28) both describe how the two-visibility correlations $V_{2}\left(\boldsymbol{U}_{a}, \nu_{i} ; \boldsymbol{U}_{b}, \nu_{j}\right)$, which can be measured directly from observations, is related to the statistics of the sky signal namely $\operatorname{MAPS} \mathcal{C}_{\ell}\left(\nu_{i}, \nu_{j}\right)$. We see that in both cases the two-visibility correlation is a weighted sum of $\mathcal{C}_{\ell}\left(\nu_{i}, \nu_{j}\right)$. We have the weights $\tilde{a}\left(\boldsymbol{U}_{a}-\boldsymbol{U}\right) \tilde{a}\left(\boldsymbol{U}_{b}-\boldsymbol{U}\right)$ in the FSA (eq. 21) where $\ell=2 \pi|\boldsymbol{U}|$. First considering a situation where $\boldsymbol{U}_{a}=\boldsymbol{U}_{b}$, the weight $\left|\tilde{a}\left(\boldsymbol{U}_{a}-\boldsymbol{U}\right)\right|^{2}$ peaks at MNRAS 000, 1-?? (2018) 
$\ell=2 \pi\left|\boldsymbol{U}_{a}\right|$ and we have a contribution from the range of multipoles $\Delta \ell \sim \pm 2 \pi D / \lambda$, where $D$ is the dimension of the antenna aperture, the weight falls to zero beyond this interval. Considering two different baselines $\boldsymbol{U}_{a} \neq \boldsymbol{U}_{b}$, the weights are the product of two functions, one which peaks at $\boldsymbol{U}=\boldsymbol{U}_{a}$ and another peaks at $\boldsymbol{U}=\boldsymbol{U}_{b}$. The weights have non-zero values only if there is some overlap between $\tilde{a}\left(\boldsymbol{U}_{a}-\boldsymbol{U}\right)$ and $\tilde{a}\left(\boldsymbol{U}_{b}-\boldsymbol{U}\right)$ i.e. $\left|\boldsymbol{U}_{a}-\boldsymbol{U}_{b}\right|<$ $2 \pi D / \lambda$. There is no overlap and the visibilities are uncorrelated if $\left|\boldsymbol{U}_{a}-\boldsymbol{U}_{b}\right|>2 \pi D / \lambda$. The properties of the FSA visibility correlation have been analyzed in detail in several earlier works (Bharadwaj \& Sethi 2001; Bharadwaj \& Ali 2005).

Considering the SH formalism (eq. 28), we see that, here also the weights are a product of two functions (eq. 29). Recollect that $B_{\ell}^{m}(\boldsymbol{U}, \nu)$ peaks at $\ell=2 \pi|\boldsymbol{U}|$ and has a width $\Delta \ell \sim \pm 2 \pi D / \lambda$ around this value. Considering the weight function for $\boldsymbol{U}_{a}=\boldsymbol{U}_{b}$ we see that this peaks at $\ell=2 \pi\left|\boldsymbol{U}_{a}\right|$, or in other words, the visibility correlation $V_{2}\left(\boldsymbol{U}_{a}, \nu_{i} ; \boldsymbol{U}_{a}, \nu_{j}\right)$ essentially responds to $\mathcal{C}_{\ell}\left(\nu_{i}, \nu_{j}\right)$ in a range of multipoles $\Delta \ell= \pm 2 \pi D / \lambda$ peaked around $\ell=2 \pi\left|\boldsymbol{U}_{a}\right|$. The fact that the index $m$ does not appear in eq. (28) is a consequence of the assumption that the sky signal is statistically homogeneous and isotropic on the sky. Next, considering two different baseline $\boldsymbol{U}_{a} \neq \boldsymbol{U}_{b}$, here again we see that the expectations are qualitatively similar to these for the FSA i.e. the visibilities are correlated only if $\left|\boldsymbol{U}_{a}-\boldsymbol{U}_{b}\right|<$ $2 \pi D / \lambda$ and there is no correlation for larger separations. Although the visibility correlations are expected to be qualitatively similar in both the FSA and the SH, the predicted values are expected to differ. We have quantified these differences in subsequent sections of this paper.

\subsection{The OWFA Window Function $\left(\mathcal{W}_{\ell}\right)$}

In this subsection we explicitly calculate the window function and discuss its behaviour for the two modes PI and PII of OWFA. We have used the OWFA aperture power pattern (eq. 2) in eq. (14) to evaluate $B_{\ell}^{m}(\boldsymbol{U}, \nu)$. Finally, we have used eq. (29) to compute the window function $\mathcal{W}_{\ell}\left(\boldsymbol{U}_{a}, \nu_{i} ; \boldsymbol{U}_{b}, \nu_{j}\right)$. For comparison, we have also used LA (eq. 16) to calculate $B_{\ell}^{m}(\boldsymbol{U}, \nu)$ and used this in eq. (29) to compute the corresponding window function $\mathcal{W}_{\ell}\left(\boldsymbol{U}_{a}, \nu_{i} ; \boldsymbol{U}_{b}, \nu_{j}\right)$. Throughout the entire subsequent analysis we have assumed that the pointing direction $\hat{\boldsymbol{p}}$ is towards the celestial equator i.e. normal to the aperture (Figure 1) which results in $e^{-2 \pi i U^{\prime} \cdot \hat{p}}=1$.

We first consider the OWFA window function $\mathcal{W}_{\ell}\left(\boldsymbol{U}_{a}, \nu_{i} ; \boldsymbol{U}_{b}, \nu_{j}\right)$ for a situation where 


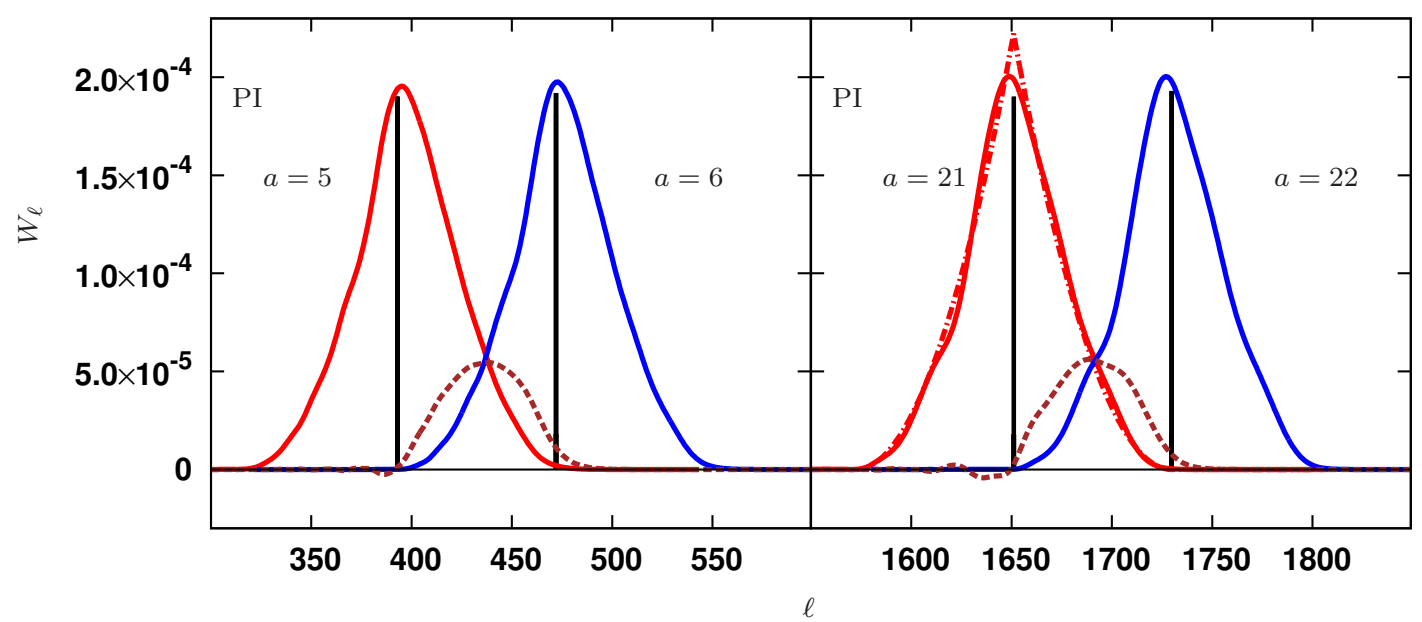

(a)

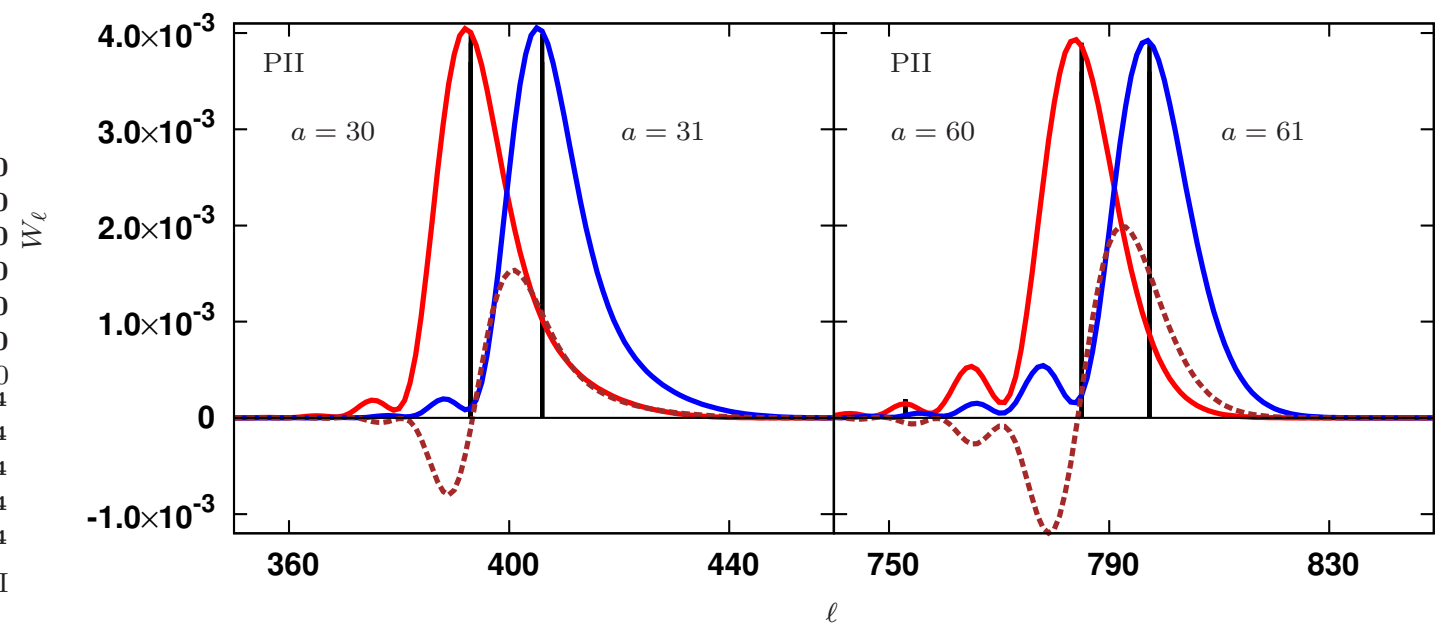

(b)

Figure 7. This shows the window functions for OWFA PI and PII. Solid lines in the top row show the window functions $\mathcal{W}_{\ell}\left(\boldsymbol{U}_{a}, \nu_{c} ; \boldsymbol{U}_{a}, \nu_{c}\right)$ for the same baselines of OWFA PI for $a=5,6,21$ and 22 . The dot-dashed line shows the $\mathcal{W}_{\ell}\left(\boldsymbol{U}_{a}, \nu_{c} ; \boldsymbol{U}_{a}, \nu_{c}\right)$ evaluated using LA for $a=21$. Solid lines in the bottom row show the window functions $\mathcal{W}_{\ell}\left(\boldsymbol{U}_{a}, \nu_{c} ; \boldsymbol{U}_{a}, \nu_{c}\right)$ for the same baselines of OWFA PII for $a=30,31,60$ and 61 . The dashed lines in the figure represents the window functions $\mathcal{W}_{\ell}\left(\boldsymbol{U}_{a}, \nu_{c} ; \boldsymbol{U}_{a \pm 1}, \nu_{c}\right)$ for the corresponding adjacent baselines.

we have the same baseline $\boldsymbol{U}_{a}=\boldsymbol{U}_{b}=a d / \lambda$. Based on our earlier discussion, the OWFA window function $\mathcal{W}_{\ell}\left(\boldsymbol{U}_{a}, \nu_{i} ; \boldsymbol{U}_{a}, \nu_{j}\right)$ is expected to peak at $\ell_{a}=2 \pi a d / \lambda$ and have a width of $\Delta \ell= \pm 2 \pi d / \lambda$ around $\ell_{a}$. Figure 7 shows the OWFA window function $\mathcal{W}_{\ell}\left(\boldsymbol{U}_{a}, \nu_{i} ; \boldsymbol{U}_{b}, \nu_{j}\right)$ as a function of $\ell$ for a few fixed values of $\boldsymbol{U}_{a}$ (mentioned in the figure legend), and fixed frequency $\nu_{i}=\nu_{j}=\nu_{c}=326.5 \mathrm{MHz}$, which is the nominal frequency for OWFA. The top row shows the results for OWFA PI where we see that $\mathcal{W}_{\ell}\left(\boldsymbol{U}_{a}, \nu_{c} ; \boldsymbol{U}_{a}, \nu_{c}\right)$ peaks at $\ell_{a}=395,471,1650$ and 1725 for $a=5,6,21$ and 22 respectively which is consistent with $\ell_{a} \approx 2 \pi a d / \lambda$ where $d=11.5 \mathrm{~m}$ and $2 \pi d / \lambda \approx 79$. We also see that, as expected, for all values of $a$ the window function extends over a nearly fixed interval of $\Delta \ell= \pm 79$ around the peak values. The dot-dashed line in the right panel of the top row shows the window function 
calculated using LA for $a=21$. We find that LA works well in the large baseline regime. The bottom row shows the results for OWFA PII where we see that $\mathcal{W}_{\ell}\left(\boldsymbol{U}_{a}, \nu_{c} ; \boldsymbol{U}_{a}, \nu_{c}\right)$ peaks at $\ell_{a}=390,405,782$ and 797 for $a=30,31,60$ and 61 respectively which is consistent with $\ell_{a} \approx 2 \pi a d / \lambda$ where $d=1.92 \mathrm{~m}$ and $2 \pi d / \lambda \approx 13.13$. Considering the width of the window function, we see that for all values of $a, \mathcal{W}_{\ell}\left(\boldsymbol{U}_{a}, \nu_{c} ; \boldsymbol{U}_{a}, \nu_{c}\right)$ goes to zero at $\Delta \ell \approx 13$ to the left of $\ell_{a}$. However, unlike PI, here we see that $\mathcal{W}_{\ell}\left(\boldsymbol{U}_{a}, \nu_{c} ; \boldsymbol{U}_{a}, \nu_{c}\right)$ shows several secondary peaks (oscillations) after the first zero to the left of $\ell_{a}$ and it shows a tail which extends to $\Delta \ell \approx 40$ to the right of $\ell_{a}$. The oscillatory features and the extended tail are both a consequence of the spherical Bessel function $j_{\ell}\left(2 \pi\left|\boldsymbol{U}^{\prime}\right|\right)$ in eq. (14). For PI these oscillations and the tail are washed out because of the integral over the broader aperture power pattern $\tilde{a}\left(\boldsymbol{U}_{a}-\boldsymbol{U}^{\prime}\right)$.

We next consider the OWFA window function $\mathcal{W}_{\ell}\left(\boldsymbol{U}_{a}, \nu_{i} ; \boldsymbol{U}_{b}, \nu_{j}\right)$ for a situation where we have the two different baselines $\boldsymbol{U}_{a} \neq \boldsymbol{U}_{b}$. The aperture power patterns $\tilde{a}\left(\boldsymbol{U}_{a}-\boldsymbol{U}\right)$ and $\tilde{a}\left(\boldsymbol{U}_{b}-\boldsymbol{U}\right)$ have an overlap only if $b=a \pm 1$ i.e. the overlap is restricted to the adjacent baselines and does not extend beyond (Figure 2 of Ali \& Bharadwaj 2014). We further expect the window function $\mathcal{W}_{\ell}\left(\boldsymbol{U}_{a}, \nu_{c} ; \boldsymbol{U}_{a \pm 1}, \nu_{c}\right)$ for the adjacent baselines to peak at $\ell_{a}=\pi(2 a \pm 1) d / \lambda$ which corresponds to the average of the two baselines and have a width $\Delta \ell= \pm \pi d / \lambda$ which is half the width of $\mathcal{W}_{\ell}\left(\boldsymbol{U}_{a}, \nu_{c} ; \boldsymbol{U}_{a}, \nu_{c}\right)$. For both PI and PII we find that the peaks of the window function $\mathcal{W}_{\ell}\left(\boldsymbol{U}_{a}, \nu_{c} ; \boldsymbol{U}_{a \pm 1}, \nu_{c}\right)$ are located at the expected $\ell$ values, the width also is consistent with the expectations for PI. However, for PII we find that $\mathcal{W}_{\ell}\left(\boldsymbol{U}_{a}, \nu_{c} ; \boldsymbol{U}_{a \pm 1}, \nu_{c}\right)$ oscillates to the left of the peak and has an extended tail to right. As mentioned earlier, these are consequences of the spherical Bessel function $j_{\ell}\left(2 \pi\left|\boldsymbol{U}^{\prime}\right|\right)$ in eq.(14). We finally note that, for both PI and PII there is an $\ell$ range where the window function $\mathcal{W}_{\ell}\left(\boldsymbol{U}_{a}, \nu_{c} ; \boldsymbol{U}_{a \pm 1}, \nu_{c}\right)$ become negative, while this is relatively prominent for PII this feature is also present for PI.

\section{SIMULATION}

In the previous section we have developed a formalism which relates the two-visibility correlations $V_{2}\left(\boldsymbol{U}_{a}, \nu_{i} ; \boldsymbol{U}_{b}, \nu_{j}\right)$, which can be measured from the observations, to the statistics of the sky signal namely MAPS $\mathcal{C}_{\ell}\left(\nu_{i}, \nu_{j}\right)$. In this section we present simulations that we have carried out to validate this theoretical formalism. The entire analysis is restricted to a 


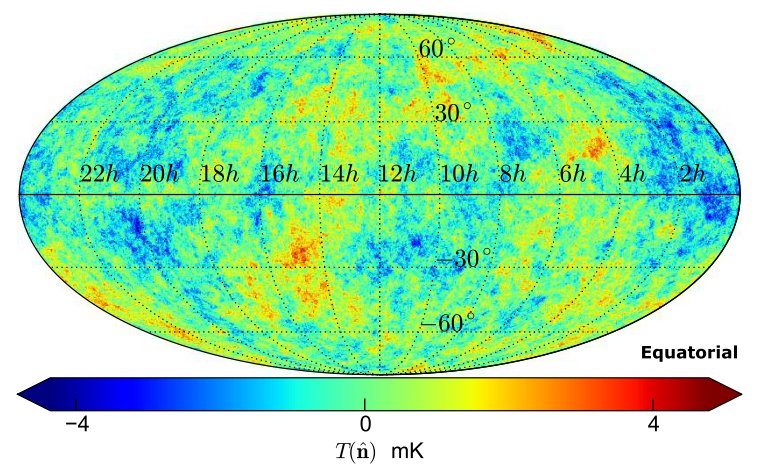

Figure 8. This shows a single realization ofthe brightness temperature fluctuations used in the simulations.

single frequency $\nu_{c}=326.5 \mathrm{MHz}$ and do not show this explicitly in much of the subsequent text.

We assume that the sky signal $T(\hat{\boldsymbol{n}})$ is a Gaussian random field with an angular power spectrum $C_{\ell}$ which we have modelled as a power law

$\mathcal{C}_{\ell}=A\left(\frac{\ell}{\ell_{0}}\right)^{n}$

arbitrarily normalized to unity (i.e. $A=1$ ) at $\ell=\ell_{0}=1$. We have considered the power law index to have value $n=-2$ in our analysis.

The simulations were carried out using the package HEALPix (Hierarchical Equal Area isoLatitude Pixelization of a sphere; Górski et al. 2005). For OWFA, the largest baseline corresponds to an angular multipole $\ell \approx 2 \pi \times 530 \mathrm{~m} / \lambda_{c}=3624$. In the simulations, we have set $\ell_{\max }=4096$ which corresponds to a pixel size of $1.712^{\prime}$. We have used the SYNFAST routine of HEALPix to generate different statistically independent realization of the sky signal $T(\hat{\boldsymbol{n}})$ corresponding to the input $C_{\ell}$.

We have computed the OWFA visibilities from the simulated maps using eq. (5). Here, it is convenient to adopt the coordinate system defined in Figure 2 of Marthi et al. (2017) whereby

$\mathcal{V}\left(\boldsymbol{U}_{a}\right)=Q \Delta \Omega_{p i x} \sum_{p=0}^{N_{p i x}-1} T\left(\alpha_{p}, \delta_{p}\right) A\left(\alpha_{p}, \delta_{p}\right) e^{-2 \pi i U_{a}\left(\sin \delta_{p}-\sin \delta_{0}\right)}$,

where $\Delta \Omega_{p i x}$ refers to the solid angle subtended by each simulation pixel, $\left(\alpha_{p}, \delta_{p}\right)$ refers to the (RA, DEC) of the $p$-th pixel, and $\left(\alpha_{0}, \delta_{0}\right)$ refers to the pointing direction. The sum here runs over all the pixels $\left(N_{p i x}\right.$ in number) in the simulation.

The OWFA primary beam pattern $A(\Delta \hat{\boldsymbol{n}})$ eq. (3) can be expressed as $A\left(\alpha_{p}, \delta_{p}\right)=\operatorname{sinc}^{2}\left(\frac{\pi b \nu_{c}}{c} \cos \delta_{p} \sin \left(\alpha_{p}-\alpha_{0}\right)\right) \operatorname{sinc}^{2}\left(\frac{\pi d \nu_{c}}{c}\left(\sin \delta_{p}-\sin \delta_{0}\right)\right)$.

As mentioned earlier, we have used $\left(\alpha_{0}, \delta_{0}\right)=(0,0)$ for the simulations presented here. 


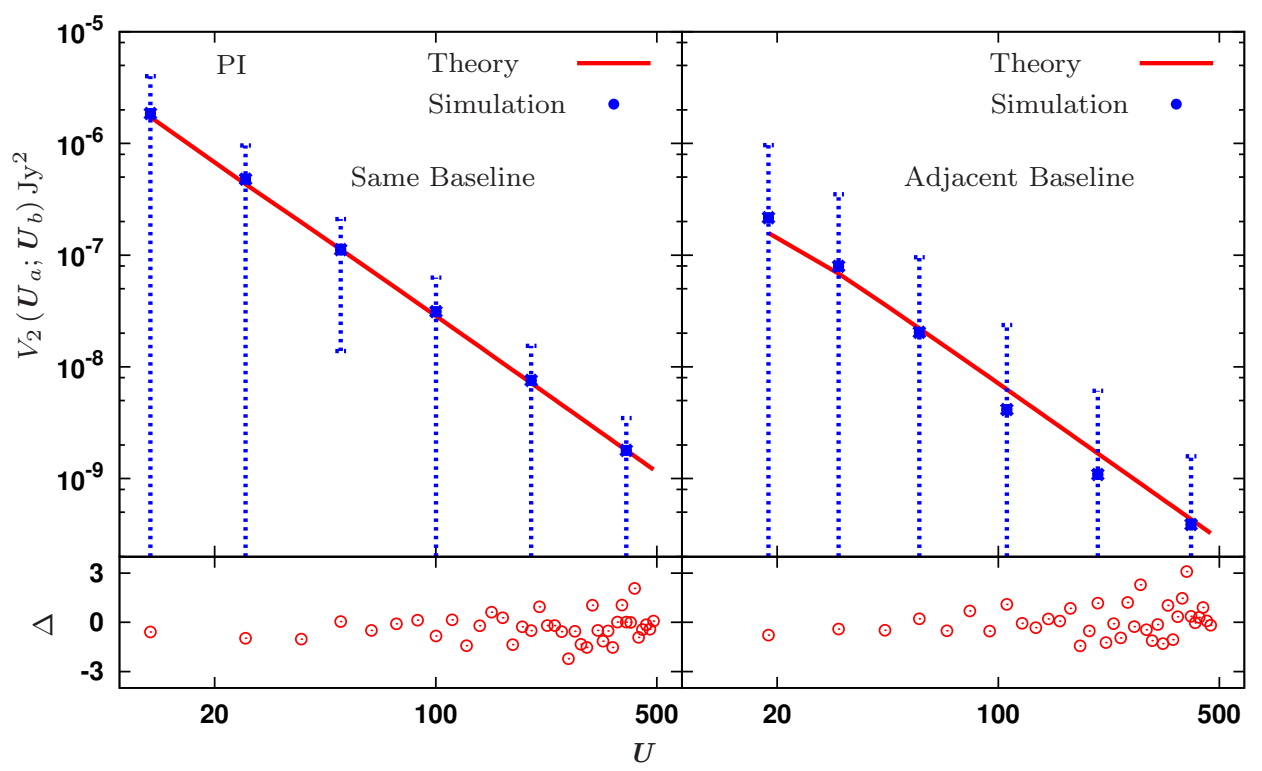

Figure 9. The upper left and right panels show the two-visibility correlation $V_{2}\left(\boldsymbol{U}_{a} ; \boldsymbol{U}_{b}\right)$ for the same and the adjacent baselines respectively for OWFA PI. The solid lines show the theoretical predictions (eq. 28) while the points with error-bars show the mean and standard deviation from the simulations considering a few selected baselines. The points in the bottom panels show $\Delta$ (eq. 33) which quantifies the deviation between the theoretical predictions and the simulations.

Figure 8 shows the brightness temperature fluctuations corresponding to a particular realization of the simulations. We have used an ensemble of 100 independent realizations of the simulated sky signal to estimate the visibility correlation $V_{2}\left(\boldsymbol{U}_{a} ; \boldsymbol{U}_{b}\right)$ and its variance.

\section{RESULTS}

The upper panels of Figure 9 show the two-visibility correlation $V_{2}\left(\boldsymbol{U}_{a} ; \boldsymbol{U}_{b}\right)$ for OWFA PI considering the fixed frequency $\nu_{i}=\nu_{j}=\nu_{c}$, which has not been shown explicitly. The left panel considers the situation where the two baselines are same $\boldsymbol{U}_{a}=\boldsymbol{U}_{b}$, whereas the right panel shows the results for the adjacent baselines $\boldsymbol{U}_{b}=\boldsymbol{U}_{a+1}$. We have used the $\mathcal{C}_{\ell}$ given by eq. (30) in eq. (28) to calculate the full $\mathrm{SH}$ theoretical predictions for the two-visibility correlations $V_{2}\left(\boldsymbol{U}_{a} ; \boldsymbol{U}_{b}\right)$ shown by the solid lines in Figure 9. We find that for both the same and the adjacent baselines, the theoretical predictions are in good agreement with the simulation. Note that $N_{r}=100$ independent realizations were used to estimate the mean $V_{2}\left(\boldsymbol{U}_{a} ; \boldsymbol{U}_{b}\right)$ (shown with points) and the standard deviation $\sigma$ (shown with error bars). The lower panels of Figure 9 show

$\Delta=\frac{\delta V_{2}\left(\boldsymbol{U}_{a} ; \boldsymbol{U}_{b}\right) \sqrt{N_{r}}}{\sigma}$ 


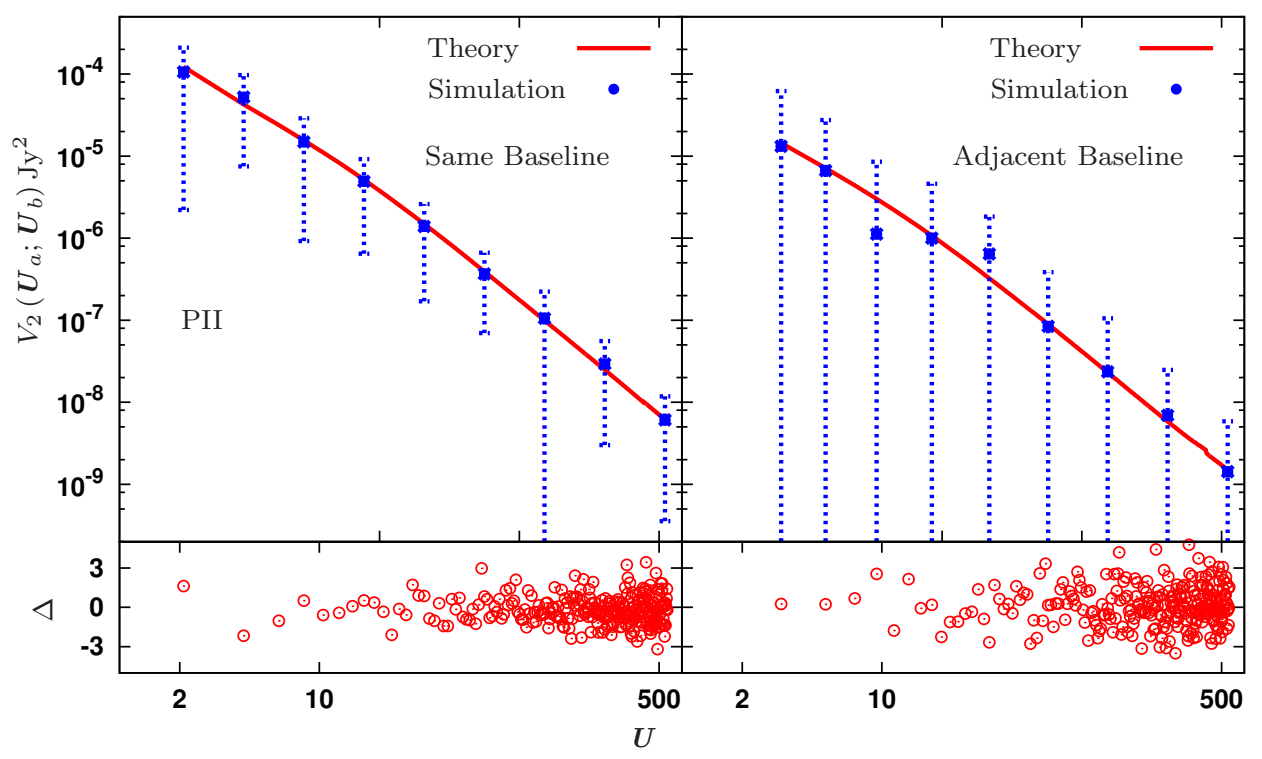

Figure 10. The same as Figure 9 for OWFA PII.

where $\delta V_{2}\left(\boldsymbol{U}_{a} ; \boldsymbol{U}_{b}\right)$ is the difference between the theoretical predictions and the simulations. We expect, $\Delta$ to have a Gaussian distribution with zero mean and unit variance. We find that the values of $\Delta$ in the lower panel of Figure 9 are roughly centred around zero and distributed within \pm 3 , consistent with what one would expect from the Gaussian distribution.

Figure 10 shows a comparison of the theoretical predictions with the simulations for the two visibility correlations for OWFA PII. Here also we find that the theoretical predictions are in good agreement with the simulations. The fact that the theoretical predictions are in good agreement with the simulations for both OWFA PI and PII validates our formalism for calculating the beam transfer function (eq. 14), the window function (eq. 29) and the visibility correlation (eq. 28).

Considering the two-visibility correlation $V_{2}\left(\boldsymbol{U}_{a} ; \boldsymbol{U}_{b}\right)$ for OWFA PI, the upper panels of Figure 11 show a comparison between the FSA (eq. 21) (solid lines), the full SH formalism (eqs. 14, 28, 29) (points), and the SH formalism with LA for $B_{\ell}^{m}(\boldsymbol{U})$ (eqs. 18, 28, 29) (dotdashed lines). The lower panels show the percentage deviation of the FSA and LA predictions relative to the full $\mathrm{SH}$ analysis. Considering the correlations at the same baseline (left panels), we find that the FSA is in good agreement with the full SH predictions over the entire $U$ range and the deviations are less than 5\%. Considering the LA, we find that the results match the full SH analysis at large baselines, the deviations are $\leqslant 5 \%$ for $U \geqslant 60$. However, these deviations increase rapidly at smaller baselines and we have $\sim 100 \%$ deviations at the 


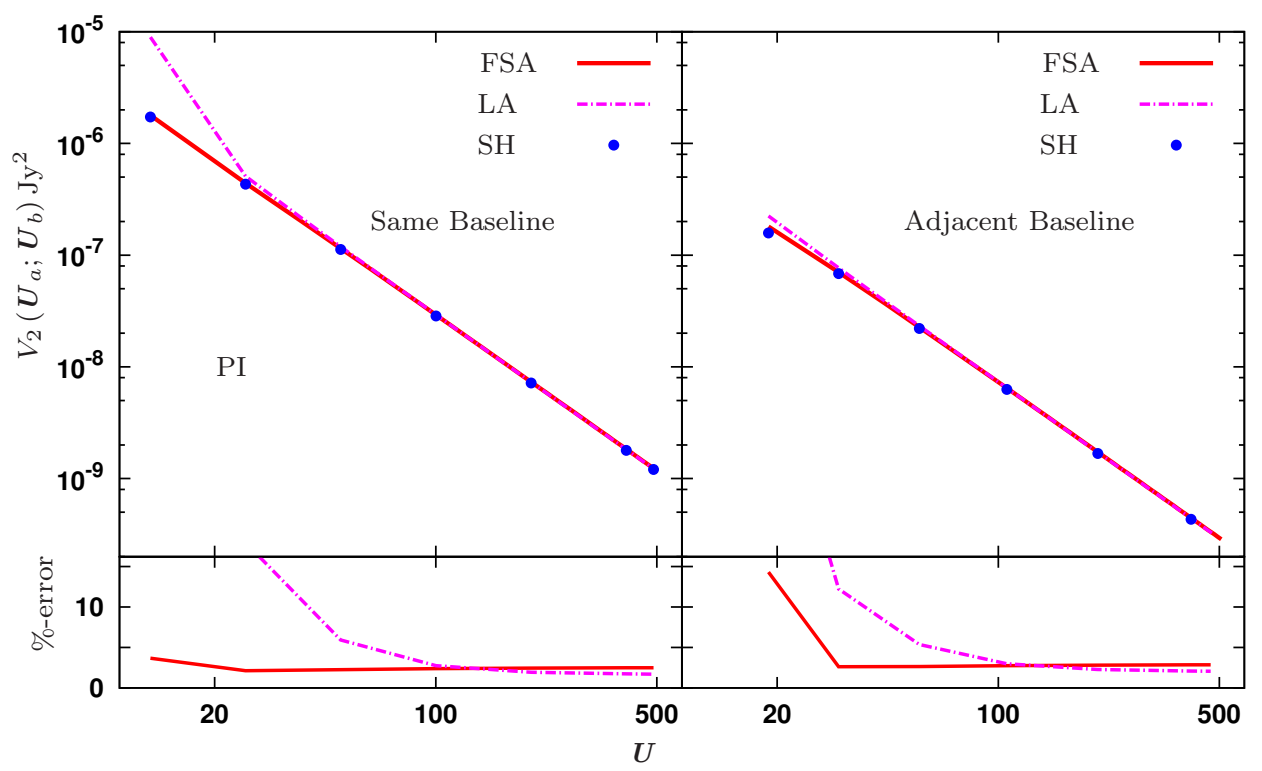

Figure 11. The upper left and right panels show the two-visibility correlation $V_{2}\left(\boldsymbol{U}_{a} ; \boldsymbol{U}_{b}\right)$ for the same and the adjacent baselines respectively for OWFA PI. The solid lines show the theoretical predictions obtained using the FSA (eq. 21), the points correspond to the predictions obtained from the full SH formalism (eqs. 14, 28, 29)and the dot-dashed lines show predictions obtained using the SH formalism with LA for $B_{\ell}^{m}(\boldsymbol{U})$ (eqs. 18, 28, 29). In the lower panels, we show the percentage deviation of the FSA (in solid lines) and LA (in dot-dashed lines)predictions relative to the full SH analysis.

smallest baseline $(U=12.5)$. For the correlations at the adjacent baselines (right panel), we find that the FSA is in good agreement with the full SH predictions for $U \geqslant 40$ and the deviation goes upto $14 \%$ at the smallest baseline $(U=18.25)$. Considering the LA, we find that the results match the full SH analysis at large baselines, the deviations are $\leqslant 5 \%$ for $U \geqslant 60$. However, these deviations increase rapidly at smaller baselines and we have $\sim 42 \%$ deviations for the correlation between the two smallest baselines which corresponds to a mean value of $U=18.25$.

Figure 12 shows a similar comparison for OWFA PII. Considering the correlations at the same baseline (left panel), we find that the FSA is in good agreement with the full SH predictions over the entire $U$ range and the deviations are less than $10 \%$. Considering the LA, we find that the results match the full $\mathrm{SH}$ analysis at large baselines, the deviations are $\leqslant 5 \%$ for $U \geqslant 60$. However, these deviations increase rapidly at smaller baselines and we have more than $200 \%$ deviations at the smallest baseline $(U=2.1)$. For the correlations at the adjacent baselines (right panel), we find that the FSA is in good agreement with the full SH predictions for $U \geqslant 60$, and the deviation goes upto $16 \%$ at the smallest baseline pair $(U=3.15)$. Considering the LA, we find that the results match the full SH analysis at large baselines, the deviations are $\leqslant 5 \%$ for $U \geqslant 60$. However, these deviations increase rapidly at smaller baselines and we have $\sim 80 \%$ deviations for the smallest baseline pair $(U=3.15)$. 


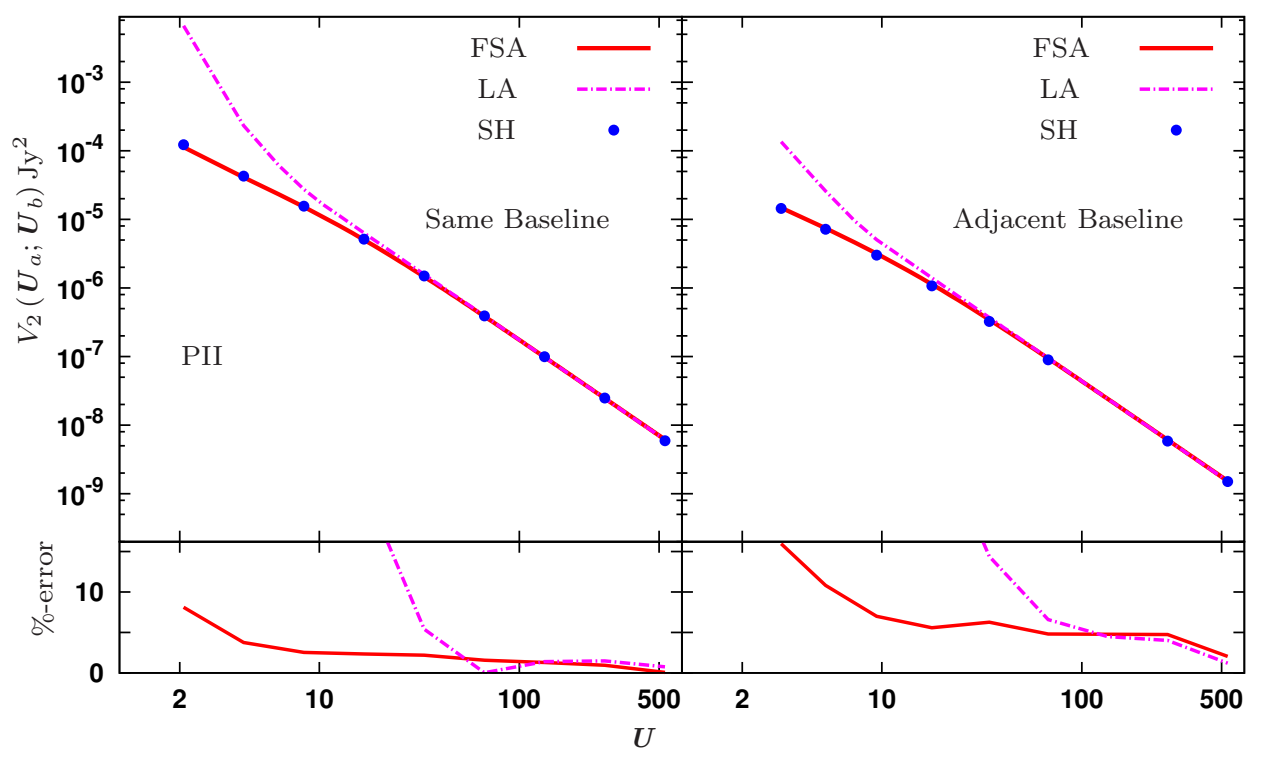

Figure 12. The same as Figure 11 for OWFA PII.

\section{SUMMARY AND CONCLUSION}

The upcoming Ooty Wide Field Array (OWFA; Subrahmanya et al. 2017a) has the detection of the redshifted 21-cm power spectrum as one of its primary science goals. Earlier works (e.g. Ali \& Bharadwaj 2014) dealing with the signal and foreground predictions for the two modes (PI and PII) of OWFA have all assumed the FSA. However, OWFA PII has a rather large FoV $\left(\sim 57^{\circ}\right)$ in the North-South direction and it is important to incorporate the spherical nature of the sky. In this paper we investigate the importance of this effect relative to the earlier FSA. To this end we adopt the $\mathrm{SH}$ analysis which relates the measured visibilities to the SH coefficients of the sky signal through a beam transfer function (Shaw et al. 2014).

Considering a radio interferometer where the baselines are all coplanar with the antenna aperture (e.g. OWFA, CHIME), we have developed a new formalism to compute the beam transfer function $B_{\ell}^{m}(\boldsymbol{U})$ as an integral of the aperture power pattern $\tilde{a}\left(\boldsymbol{U}-\boldsymbol{U}^{\prime}\right)$ (eq. 14). In addition to computational advantages, our formalism provides insight into the behaviour of $B_{\ell}^{m}(\boldsymbol{U})$, i.e. the $\ell$ and $m$ values at which we expect this to peak for a particular baseline $\boldsymbol{U}$. For OWFA, we find that $B_{\ell}^{m}(\boldsymbol{U})$ is expected to peak at $\ell \approx 2 \pi U$ and fall off with increasing $|\Delta \ell|$ with a width $\Delta \ell \approx \pm 2 \pi d / \lambda$ around the peak value. We also provide a closed form analytical expression for $B_{\ell}^{m}(\boldsymbol{U})$ using the LA which is expected to hold at large $\ell$ or equivalently at large $U$. This analytic expression (eq. 18) indicates that $B_{\ell}^{m}(\boldsymbol{U})$ is expected to peak at $m=0$ and fall off with increasing $m$ with a width $\Delta m \approx \pm(2 \ell+1) \lambda / b$ (Figure 
$5)$. It is worth noting that while $m$ takes values in the range $-\ell \leqslant m \leqslant \ell$, the sky signal is restricted to a range $-\ell \lambda / b \leqslant m \leqslant \ell \lambda / b$ where $\lambda / b \approx 0.03$ for OWFA i.e. OWFA only responds to $3 \%$ of the available $m$-modes because of the relatively large width $b$ of the telescope aperture. Figure 6 shows $B_{\ell}^{m}(\boldsymbol{U})$ for a few of the baselines. For both PI and PII we find that the $\ell$ value where $B_{\ell}^{m}(\boldsymbol{U})$ peaks is roughly consistent with the expected values, for PI the width also is consistent with this. However for PII $B_{\ell}^{m}(\boldsymbol{U})$ oscillates to the left of the peak and has an extended tail to the right of the peak. The LA provides a reasonable match for PI, particularly at large $U$. For PII, LA correctly predicts the peak, however, it does not provide a good match to the behaviour of $B_{\ell}^{m}(\boldsymbol{U})$ away from the peak even at large $U$.

In the FSA, the two-visibility correlation provides a direct estimate of the redshifted 21-cm power spectrum (Bharadwaj \& Sethi 2001; Bharadwaj \& Ali 2005). In this paper we incorporate the spherical sky and express the two-visibility correlation (eq. 28) as a weighted sum of the multi-frequency angular power spectrum (MAPS; Datta et al. 2007). The weights here are given by the window functions $\mathcal{W}_{\ell}\left(\boldsymbol{U}_{a}, \nu_{i} ; \boldsymbol{U}_{b}, \nu_{j}\right)$ which are a sum (over $m$ ) of products of the beam transfer function (eq. 29). The signal, for OWFA, is present in the correlations at the same baseline and the adjacent baselines only (Ali \& Bharadwaj 2014). Figure 7 shows the window function for a few select baselines. We see that the correlation at baseline $U_{a}$ pick up the signal corresponding to $\ell \approx 2 \pi U_{a}$ whereas the correlation between

the adjacent baselines $U_{a}$ and $U_{b}$ corresponds to $\ell \approx \pi\left(U_{a}+U_{b}\right)$. The LA provides a good match to the window function at large baselines for PI, however the match (not shown in the Figure) is not very good for PII.

We have carried out simulations to validate our spherical sky formalism. We find (Figures 9 and 10) that for both PI and PII our analytical predictions are consistent with the results from the simulations, thereby validating our formalism. Figures 11 and 12 show a comparison between the predictions of our SH analysis with those from the FSA. The situation where LA has been used to calculate the beam transfer function is also considered for comparison. We find that for both PI and PII, the FSA matches the SH predictions to within $16 \%$ across the entire baseline range. As expected, the match is better for PI as compared to PII. The match is also better for the correlations at the same baseline as compared to the adjacent baselines. In contrast, the LA provides a good match $<5 \%$ only at the large baselines $U \geqslant 60$. In conclusion, we note that the flat sky approximation matches the full $\mathrm{SH}$ analysis to within $15-16 \%$. It is necessary to use the latter if an accuracy higher than this is required. 
Finally, we note that the entire analysis here is restricted to observations in a single FoV. This has advantage in allowing detailed foreground modelling of the particular field which may be useful in foreground removal. In contrast, the " $m$-mode" analysis proposed by Shaw et al. (2014) considers drift scan observations which cover a large fraction of the sky. The latter increases the available signal, this could however make detailed foreground modelling more difficult. We plan to study $m$-mode analysis in the context of OWFA in subsequent work.

\section{ACKNOWLEDGEMENT}

The authors would like to thank Jayaram N Chengalur and Visweshwar Ram Marthi for their useful suggestions and discussions. SC acknowledges the University Grant Commission, India for providing financial support through Senior Research Fellowship. SC would also like to thank Sukannya Bhattacharya, Abinash Kumar Shaw, Anjan Kumar Sarkar and Debanjan Sarkar for their help and useful discussions.

\section{REFERENCES}

Ali S. S., Bharadwaj S., 2014, J. Astrophys. Astron., 35, 157

Ali S. S., Bharadwaj S., Pandey B., 2005, Monthly Notices of the Royal Astronomical Society, 363, 1, 251

Ali Z. S., Parsons A. R., Zheng H., et al., 2015, ApJ, 809, 61

Bandura K., Addison G. E., Amiri M., et al., 2014, in Ground-based and Airborne Telescopes V, vol. 9145 of Proc. SPIE, 914522

Bharadwaj S., Ali S. S., 2005, MNRAS, 356, 1519

Bharadwaj S., Nath B. B., Sethi S. K., 2001, J. Astrophys. Astron., 22, 21

Bharadwaj S., Pandey S. K., 2003, Journal of Astrophysics and Astronomy, 24, 23

Bharadwaj S., Sarkar A. K., Ali S. S., 2015, J. Astrophys. Astron., 36, 385

Bharadwaj S., Sethi S. K., 2001, Journal of Astrophysics and Astronomy, 22, 293

Bharadwaj S., Sethi S. K., Saini T. D., 2009, Phys. Rev. D, 79, 8, 083538

Bull P., Ferreira P. G., Patel P., Santos M. G., 2015, ApJ, 803, 21

Chang T.-C., Pen U.-L., Bandura K., Peterson J. B., 2010, Nature, 466, 463

Chang T.-C., Pen U.-L., Peterson J. B., McDonald P., 2008, Physical Review Letters, 100, 9, 091303

Chatterjee S., Bharadwaj S., Marthi V. R., 2017, Journal of Astrophysics and Astronomy, 38, 1, 15

Chen X., 2012, International Journal of Modern Physics Conference Series, 12, 256

Chen X., 2015, IAU General Assembly, 22, 2252187

Chengalur J. N., Gupta Y., Dwarkanath K., 2007, Low Frequency Radio Astronomy 3rd edition, NCRA-TIFR, India

Datta K. K., Choudhury T. R., Bharadwaj S., 2007, MNRAS, 378, 119

Ghosh A., Mertens F. G., Koopmans L. V. E., 2018, Monthly Notices of the Royal Astronomical Society, 474, 4, 4552

Gupta Y., Ajithkumar B., Kale H., et al., 2017, CURRENT SCIENCE, 113, 4, 707

Górski K. M., Hivon E., Banday A. J., et al., 2005, The Astrophysical Journal, 622, 2, 759

MNRAS 000, 1-?? (2018) 
Hazra D. K., Sarkar T. G., 2012, Physical review letters, 109, 12, 121301

Limber D. N., 1954, ApJ, 119, 655

Liu A., Zhang Y., Parsons A. R., 2016, ApJ, 833, 242

Marthi V. R., Chatterjee S., Chengalur J. N., Bharadwaj S., 2017, MNRAS, 471, 3112

Masui K. W., McDonald P., Pen U.-L., 2010, Phys. Rev. D, 81, 10, 103527

Morales M. F., Hewitt J., 2004, ApJ, 615, 7

Newburgh L., Bandura K., Bucher M., et al., 2016, arXiv preprint arXiv:1607.02059

Perley R. A., Schwab F. R., Bridle A. H., 1989

Sarkar A. K., Bharadwaj S., Marthi V. R., 2018, Monthly Notices of the Royal Astronomical Society, 473, 1, 261

Seo H.-J., Dodelson S., Marriner J., et al., 2010, ApJ, 721, 164

Shaw J. R., Sigurdson K., Pen U.-L., Stebbins A., Sitwell M., 2014, ApJ, 781, 57

Shaw J. R., Sigurdson K., Sitwell M., Stebbins A., Pen U.-L., 2015, Phys. Rev. D, 91, 8, 083514

Subrahmanya C. R., Manoharan P. K., Chengalur J. N., 2017a, J. Astrophys. Astron., 38, 10

Subrahmanya C. R., Prasad P., Girish B. S., Somashekar R., Manoharan P. K., Mittal A. K., 2017b, Journal of Astrophysics and Astronomy, 38, 1, 11

Swarup G., Ananthakrishnan S., Kapahi V. K., Rao A. P., Subrahmanya C. R., Kulkarni V. K., 1991, Current Science, Vol. 60, NO.2/JAN25, P. 95, 1991, 60, 95

Swarup G., Sarma N. V. G., Joshi M. N., et al., 1971, Nature Physical Science, 230, 185

Visbal E., Loeb A., Wyithe S., 2009, J. Cosmology Astropart. Phys., 10, 030

Wyithe J. S. B., Loeb A., Geil P. M., 2008, MNRAS, 383, 1195

Zhang J., Ansari R., Chen X., Campagne J.-E., Magneville C., Wu F., 2016a, Mon. Not. Roy. Astron. Soc., 461, 2, 1950

Zhang J., Zuo S.-F., Ansari R., et al., 2016b, Research in Astronomy and Astrophysics, 16, 10, 158

Zheng H., Tegmark M., Buza V., et al., 2014, MNRAS, 445, 1084 